OPEN ACCESS

Edited by:

Xin Qi,

Case Western Reserve University, United States

Reviewed by: Sabzali Javadov, University of Puerto Rico, Puerto Rico Kah-Leong Lim, National University of Singapore, Singapore

Louis-Eric Trudeau Université de Montréal, Canada

*Correspondence: James H. Eubanks jeubanks@uhnres.utoronto.ca

Received: 26 March 2018 Accepted: 17 June 2018 Published: 25 July 2018

Citation: Sidorova-Darmos E, Sommer $R$ and Eubanks JH (2018) The Role of SIRT3 in the Brain Under Physiological and Pathological Conditions.

Front. Cell. Neurosci. 12:196. doi: 10.3389/fncel.2018.00196

\section{The Role of SIRT3 in the Brain Under Physiological and Pathological Conditions}

\author{
Elena Sidorova-Darmos ${ }^{1,2,3}$, Rosa Sommer ${ }^{1,4}$ and James H. Eubanks 1,2,3,5* \\ ${ }^{1}$ Division of Genetics and Development, Krembil Research Institute, University Health Network, Toronto, ON, Canada, \\ ${ }^{2}$ Department of Physiology, University of Toronto, Toronto, ON, Canada, ${ }^{3}$ Institute of Medical Sciences, University of Toronto, \\ Toronto, ON, Canada, ${ }^{4}$ Department of Pharmacology and Toxicology, University of Toronto, Toronto, ON, Canada, \\ ${ }^{5}$ Department of Surgery (Neurosurgery), University of Toronto, Toronto, ON, Canada
}

Sirtuin enzymes are a family of highly seven conserved protein deacetylases, namely SIRT1 through SIRT7, whose enzymatic activities require the cofactor nicotinamide adenine dinucleotide $\left(\mathrm{NAD}^{+}\right)$. Sirtuins reside in different compartments within cells, and their activities have been shown to regulate a number of cellular pathways involved in but not limited to stress management, apoptosis and inflammatory responses. Given the importance of mitochondrial functional state in neurodegenerative conditions, the mitochondrial SIRT3 sirtuin, which is the primary deacetylase within mitochondria, has garnered considerable recent attention. It is now clear that SIRT3 plays a major role in regulating a host of mitochondrial molecular cascades that can contribute to both normal and pathophysiological processes. However, most of the currently available knowledge on SIRT3 stems from studies in non-neuronal cells, and the consequences of the interactions between SIRT3 and its targets in the CNS are only beginning to be elucidated. In this review, we will summarize current advances relating to SIRT3, and explore how its known functions could influence brain physiology.

Keywords: SIRT3, mitochondria, oxidative stress, neurodegenerative disease, pharmacology

\section{INTRODUCTION}

It has been over three decades since the silent information regulator 2 (Sir2) gene was initially identified in yeast (Shore et al., 1984; Ivy et al., 1986; Rine and Herskowitz, 1987; Tanner et al., 2000) and its product shown to regulate DNA recombination/repair, gene silencing and chromosomal stability (Brachmann et al., 1995; Kaeberlein et al., 1999). Interest in SIR2 increased substantially when longevity-enhancements were associated with its over-expression in Saccharomyces cerevisiae (Kaeberlein et al., 1999; Kim et al., 1999; Wood et al., 2004), C. elegans (Tissenbaum and Guarente, 2001) and Drosophila melanogaster (Wood et al., 2004), and the identification of seven SIR2 homologs (SIRT1-7) in mammalian systems (Brachmann et al., 1995; Frye, 2000; Finkel et al., 2009) only added further interest to the family. Unlike the restricted nuclear localization of the ancestral SIR2 protein in yeast, however, specific mammalian sirtuins localize within different sub-cellular compartments. Like the ancestral Sir2, sirtuins SIRT1, SIRT6 and SIRT7 reside predominantly within the nucleus (Michishita et al., 2005). SIRT2 is largely cytosolic (Perrod et al., 2001; North et al., 2003), while sirtuins SIRT3, SIRT4 and SIRT5 are primarily found within mitochondria (Onyango et al., 2002; Ahuja et al., 2007; Nakamura et al., 2008). Defining 
the role played by each individual sirtuin within these specific regions remains a major topic of investigation. Structurally, while each member of the sirtuin family possesses a conserved catalytic core domain and a nicotinamide adenine dinucleotide $\left(\mathrm{NAD}^{+}\right)$binding motif, each member displays distinct $\mathrm{N}$ - and C-terminal regions that contribute to their localization and functional specificities (Landry et al., 2000; Min et al., 2001; Marmorstein, 2004). While other enzymatic activities have been reported for some members of the family (see Table 1, Du et al., 2011; Peng et al., 2011; Jiang et al., 2013; Bao et al., 2014; Mathias et al., 2014; Tan et al., 2014), $\mathrm{NAD}^{+}$-dependent protein deacetylase and/or as a mono-[ADP-ribosyl] transferase activity appears to be the primary enzymatic function for the different sirtuins (Frye, 1999; Schwer et al., 2002; North et al., 2003; Liszt et al., 2005; Michishita et al., 2005; Mostoslavsky et al., 2006; Ahuja et al., 2007; Schlicker et al., 2008; Vakhrusheva et al., 2008; see Table 1). Due to their dependence on intracellular $\mathrm{NAD}^{+}$levels, the family of sirtuins has been hypothesized to act as key rheostats that collectively monitor and maintain the homeostatic balance of the intracellular environment (Kupis et al., 2016).

Perturbations in mitochondrial homeostasis are hypothesized to play pathogenic roles in a number of neurological diseases and conditions (Martin, 2012; Morris and Berk, 2015). Normal mitochondrial homeostasis is achieved by maintaining the proper complement and levels of mitochondrial proteins and substrates, and by governing the functional activity of these proteins by post-translational modification (Stram and Payne, 2016). One of the most common protein post-translational modifications in the mitochondria proteome is lysine acetylation, with over $65 \%$ of mitochondrial proteins being subject to acetyl-lysine regulation (Hebert et al., 2013). The SIRT3 sirtuin plays a key role in this regulation, as altered mitochondrial proteome hyperacetylation has been clearly observed in tissues from SIRT3 knockout (KO) mice, with little to no changes in mitochondrial protein acetylation observed in the same tissues from SIRT4 or SIRT5-KO mice (Lombard et al., 2007). As post-translational protein acetylation is a modification that can dramatically influence the enzymatic

\begin{tabular}{ll}
\hline TABLE 1 | Mechanisms of action for sirtuin family members. \\
\hline Sirtuin & Catalytic Activities \\
\hline SIRT1 & Deacetylase (Michishita et al., 2005), \\
& Decrotonylase (Bao et al., 2014) \\
SIRT2 & Deacetylase (Perrod et al., 2001; North et al., 2003), \\
& Decrotonylase (Bao et al., 2014) \\
SIRT3 & Deacetylase (Schwer et al., 2002), \\
& Decrotonylase (Bao et al., 2014) \\
SIRT4 & ADP-ribosyltransferase (Ahuja et al., 2007), \\
& Lipoamidase (Mathias et al., 2014) \\
SIRT5 & Deacetylase (Schlicker et al., 2008) \\
& Demalonylase (Du et al., 2011; Peng et al., 2011), \\
& Desuccinylase (Du et al., 2011; Peng et al., 2011) \\
& Glutarylate (Tan et al., 2014) \\
SIRT6 & ADP-ribosyltransferase (Liszt et al., 2005); \\
& Deacetylase (Michishita et al., 2008), \\
& Acylate (Jiang et al., 2013) \\
SIRT7 & Deacetylase (Vakhrusheva et al., 2008)
\end{tabular}

activity of target proteins, these data support the hypothesis that SIRT3 serves as a key rheostat that maintains cellular metabolic homeostasis by altering the acetylation state of the mitochondrial proteome in response to changes in cellular demands (Hirschey et al., 2010; Tao et al., 2010; Cheng et al., 2016). As the brain has a particularly high aerobic metabolic rate and consumes proportionally large amounts of ATP (Falkowska et al., 2015), it is understandable why there is an increasing interest to better understand the regulation of SIRT3 expression and functional activity in nervous system tissues.

\section{SIRT3 EXPRESSION IN THE BRAIN}

Expression analysis has revealed SIRT3 to be preferentially expressed in tissues with high oxidative capacity such as brain, cardiac, hepatic, brown adipose tissue and skeletal muscle, with lower levels of expression found in tissues with lowermetabolic demand such as white adipose tissue, lungs, spleen, thymus, pancreas and small intestine (Shi et al., 2005; Lombard et al., 2007; Palacios et al., 2009; Sidorova-Darmos et al., 2014; Braidy et al., 2015). At the mRNA level, Sirt3 is the third highest expressed of all the sirtuins in the adult rat brain (behind Sirt2 and Sirt5; Sidorova-Darmos et al., 2014). High expression of SIRT3 in the CNS is not surprising, given its role in regulating mitochondrial function, metabolism and in regulating oxidative defense systems. At the protein level, SIRT3 is expressed at similar levels in the adult rat cortex, hippocampus, striatum, spinal cord, and brain stem, but is expressed at lower levels in the cerebellum (Sidorova-Darmos et al., 2014). The lower SIRT3 protein levels in cerebellum may relate to the lower number of mitochondria present in cerebellum relative to other brain regions (Frahm et al., 2005; McInerny et al., 2009; Fuke et al., 2011). SIRT3 expression within the brain is not restricted to neurons, as clear SIRT3 expression has been demonstrated in astrocytes and microglia (Kim et al., 2011; Weir et al., 2012; Dai et al., 2014; Sidorova-Darmos et al., 2014; Cheng et al., 2016). However, the relative prevalence of SIRT3 is higher in cortical neurons than in astrocytes at both the mRNA and protein levels (Sidorova-Darmos et al., 2014).

The expression profile of SIRT3 in the brain is also developmentally regulated. At the mRNA level Sirt3 expression is fairly consistent from embryonic day 18 until 24 months of age in the rat cortex, cerebellum and hippocampus (SidorovaDarmos et al., 2014). A different expression pattern is seen for SIRT3 protein during development, however, as in the rat cortex, hippocampus and cerebellum, SIRT3 protein levels increase significantly between post-natal day 7 and post-natal day 21 (Sidorova-Darmos et al., 2014). Because mitochondria play a central role in energy supply and are major regulators of neural development (Xavier et al., 2016), the observed elevation in SIRT3 levels correlates with times of increased energy demand in the central nervous system due to cellular/synaptic growth and maturation. Studies examining SIRT3 expression levels in aged brains have yielded somewhat conflicting results, however. A decrease in SIRT3 protein levels was reported in the 
18 month-old rat auditory cortex relative to 4 month-old rats (Zeng et al., 2014), and in 24 month-old rat hippocampus and frontal lobe relative to the same regions in three month-old rats (Braidy et al., 2015). However, Sidorova-Darmos et al. (2014) reported SIRT3 levels remain relatively consistent between postnatal day 21 and 24 months in the rat hippocampus and cortex, and that its expression levels increase modestly in the cerebellum between these times. The reason for this seeming discrepancy is not clear, and could potentially relate to strain and/or housing condition differences. Collectively though, these studies do show that SIRT3 is expressed in different cell lineages of the brain from early development and maintained until elderly stages, and therefore allow one to explore how drugs selectively targeting SIRT3 could influence brain function throughout life.

\section{SIRT3 PROTEIN IS EXPRESSED AS A PRECURSOR AND TRAFFICKED TO MITOCHONDRIA}

Assessments of endogenous SIRT3 localization have largely demonstrated a mitochondrial residence for SIRT3 in cells from different species (Schwer et al., 2002; Lombard et al., 2007; Scher et al., 2007). SIRT3 is expressed initially as a precursor protein of approximately 45 kilodaltons that contains a mitochondrial localization sequence (Onyango et al., 2002). Upon entry into the mitochondrial matrix, this pro-form of SIRT3 is processed into a shorter form by mitochondrial matrix processing peptidase (MMP; Onyango et al., 2002; Schwer et al., 2002). Recently, it was reported that this MMP-generated product is further cleaved by mitochondrial intermediate peptidase (MIPEP) to generate the mature enzymatically active 28 kilodalton form of SIRT3 present in the mitochondrial matrix (Kobayashi et al., 2017). However, while ubiquitously agreed that SIRT3 does reside within mitochondria, there remains debate regarding whether or not a population of some form of SIRT3 may also reside in nucleus or cytoplasm (Scher et al., 2007; Nakamura et al., 2008; Sundaresan et al., 2008). Indeed, nuclear localization of the endogenous SIRT3 precursor has been reported in U2OS and HeLa cells by immunocytochemical analysis (Scher et al., 2007; Iwahara et al., 2012) and both nuclear localization and nuclear deacetylase activity has been reported in HeLa cells and rat cardiomyocytes ectopically expressing a full-length human SIRT3 cDNA (Scher et al., 2007; Sundaresan et al., 2008). However, Cooper and Spelbrink (2008) failed to detect any nuclear SIRT3 using stringent subcellular fractionation techniques in HeLa, U2OS or HEK-293 cells that ectopically expressed a full-length human SIRT3 cDNA. Onyango et al. (2002) reported no detectable nuclear signal in COS7 cells ectopically expressing a SIRT3-GFP reporter system and a lack of endogenous SIRT3 was observed in nuclear extracts isolated from rat skeletal muscle (Gurd et al., 2012). Thus, while it is clear that the processed form of catalytically active SIRT3 resides within the mitochondria where it targets a number of mitochondrial proteins (reviewed in Bell and Guarente, 2011; Hirschey et al., 2011; Bause and Haigis, 2013; Ansari et al., 2017), it remains controversial whether additional subcellular expression patterns and non-mitochondrial protein targets exist for SIRT3 under normal conditions.

\section{SIRT3 FUNCTION IN MITOCHONDRIA}

SIRT3 is the primary sirtuin deacetylase in mitochondria. SIRT3-KO mice display globally elevated mitochondrial acetylation levels in liver, while SIRT4 or SIRT5 KO mice show no changes in mitochondrial proteome acetylation (Lombard et al., 2007). Moreover, a hyperacetylation of mitochondrial proteins was observed in myocardial tissue following infarction, and this change coincided with a downregulation of SIRT3 expression with both SIRT4 and SIRT5 expression levels being unaffected (Parodi-Rullan et al., 2012). It is important to note, however, that the role of SIRT4 and SIRT5 in mitochondria can provide benefit independent from SIRT3. For example, over-expression of SIRT4 markedly protected $\mathrm{H} 9 \mathrm{c} 2$ cardiomyoblast cells from apoptosis following hypoxia (Liu et al., 2013), and SIRT5 over-expression in SH-EP neuroblastoma cells decreased apoptosis and oxidative stress induced by staurosporine and hydrogen peroxide, respectively (Liang et al., 2017). While a substantial number of mitochondrial SIRT3 substrates have been identified (see below), only a handful of SIRT4 and SIRT5 targets have thus far been uncovered (reviewed in Parihar et al., 2015). This is an area of growing interest, as cooperation between the three mitochondrial sirtuins may be required to maintain proper homeostatic balance within the mitochondria-both under normal and pathogenic circumstances.

While there have been fewer studies of SIRT3 activity in neural cells or tissues, studies employing non-neuronal systems have uncovered a number of mitochondrial proteins targeted by SIRT3 that are also expressed in brain, and therefore it seems likely they will be targeted similarly by SIRT3 in nervous system settings. These include members of pathways involved in anti-oxidative defense systems (Jacobs et al., 2008; Tao et al., 2010; Bell and Guarente, 2011; Chen et al., 2011; Ren et al., 2016), metabolism (Hallows et al., 2006; Ahn et al., 2008; Schlicker et al., 2008; Salvatori et al., 2017), and mitochondrial biogenesis (Shi et al., 2005; Kong et al., 2010; Yang et al., 2010; Giralt et al., 2011; Dai et al., 2014; Zhao et al., 2016). Visual overview of some of these pathways is outlined in Figure 1. Each of these pathways has direct relevance to brain physiology, and alterations in the function of each have been implicated as contributing factors in different neurodegenerative diseases or neurological disorders (Federico et al., 2012; Guo et al., 2013; Kim et al., 2015; Li et al., 2017).

\section{SIRT3 Regulation of Anti-Oxidative Defense Systems}

Of particular interest to neural cells, SIRT3 deacetylates and increases the catalytic activity of manganese superoxide dismutase (MnSOD; Qiu et al., 2010; Tao et al., 2010; Chen et al., 2011; Shi et al., 2017), which is the primary mitochondrial enzyme that converts $\mathrm{O}_{2}{ }^{-}$to $\mathrm{H}_{2} \mathrm{O}_{2}$, and the TCA cycle enzyme isocitrate dehydrogenase (IDH2; Schlicker et al., 2008; Someya et al., 2010; Yu et al., 2012), which converts $\mathrm{NADP}^{+}$to $\mathrm{NADPH}$ 


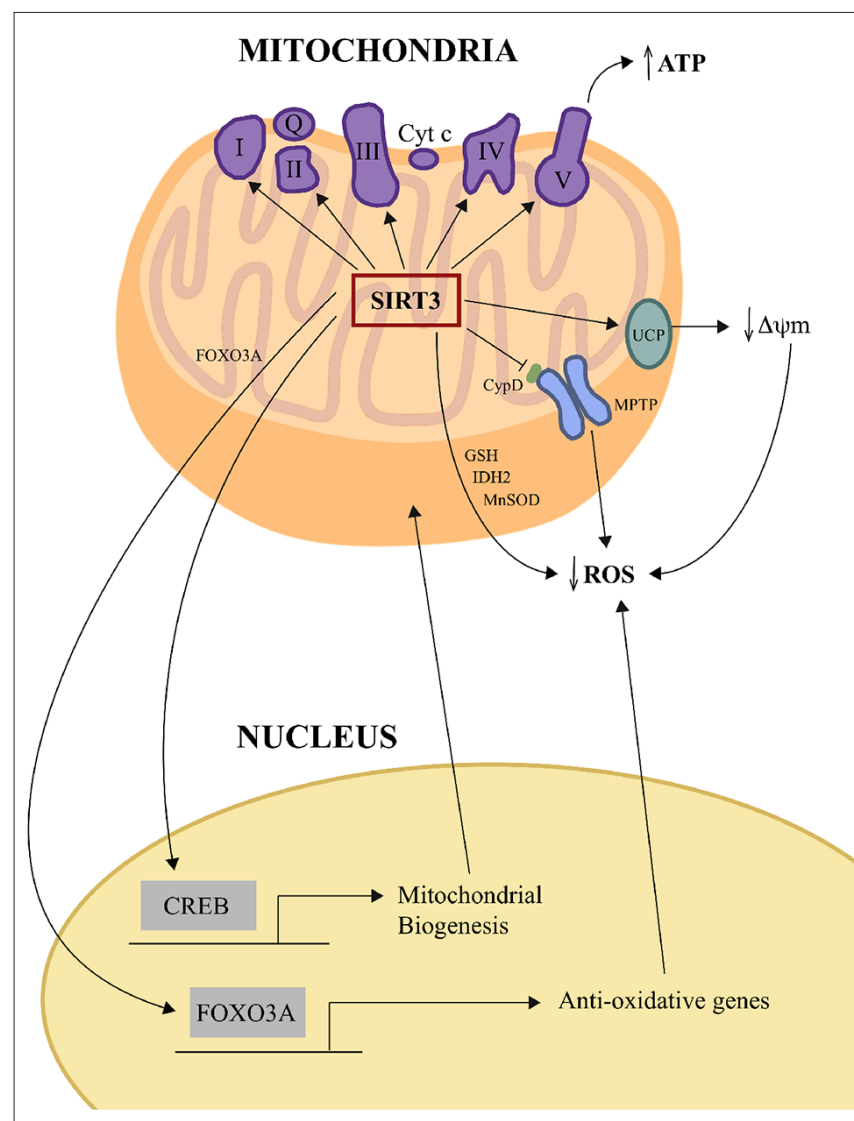

FIGURE 1 | SIRT3 influences oxidative phosphorylation and oxidative stress management. SIRT3 increases ETC efficiency by deacetylating and regulating the activities of mitochondrial complexes I, II, III, IV and V to increase ATP production. SIRT3 reduces mitochondrial oxidative stress directly by deacetylating IDH2 and MnSOD and also increasing GSH levels, and thereby enhancing ROS detoxification. SIRT3 can also negatively regulate ROS levels in some tissues indirectly by upregulating UCP expression, which lessens the driving force for mitochondrial ROS production by decreasing $\Delta \Psi \mathrm{m}$. SIRT3 can also negatively regulate the opening of the MPTP by deacetylating CypD. SIRT3 also orchestrates mitochondria-nuclear cross-talk by deacetylating and activating mitochondrial FOXO3A, which then travels to the nucleus to activate the transcription of anti-oxidative genes, and by indirectly activating the transcription factor CREB, which activates genes whose products play roles in mitochondrial biogenesis. CREB, cAMP response element-binding protein; CypD, cyclophilin D; ETC, electric transport chain; FOXO3a, forkhead box O3; GSH, glutathione; IDH2, isocitrate dehydrogenase; MnSOD, manganese superoxide dismutase; MPTP mitochondrial permeability transition pore; ROS, reactive oxygen species; UCP, uncoupling protein; $\Delta \Psi \mathrm{m}$, change in mitochondrial membrane potential.

in mitochondria and helps maintain the anti-oxidant glutathione (GSH) in a reduced and active state. SIRT3 also physically interacts with thioredoxin-2 (Palacios et al., 2009), which is a member of the thioredoxin system and also involved in $\mathrm{H}_{2} \mathrm{O}_{2}$ scavenging. Indeed, converging studies show that cells and tissues deficient in SIRT3 show high indices of oxidative stress (reviewed in Bell and Guarente, 2011; Bause and Haigis, 2013; Ansari et al., 2017). Interestingly Parodi-Rullam et al. (2017) observed no difference in oxidative stress between WT and SIRT3-KO mouse hearts under basal conditions. Because there was an increase in SIRT4 expression in SIRT3-KO hearts, these authors suggested that SIRT4 may serve as an independent anti-oxidative compensatory mechanism in these mice under normal conditions. However, following ischemia-reperfusion injury, SIRT3-deficient heart mitochondria showed elevations of reactive oxygen species (ROS) and oxidative damage relative to WT mitochondria, illustrating any potential compensatory SIRT4 activity has a ceiling relative to that of SIRT3. Future studies will be required to define the interplay between mitochondrial sirtuins and regulation of anti-oxidative systems in mitochondria under basal and various pathological conditions. Nevertheless, these results strongly suggest SIRT3 likely plays a key role in regulating ROS levels in neurons, where aerobic respiration is the primary mechanism for energy production.

\section{SIRT3 Influence on Mitochondrial Membrane Potential and ROS Genesis}

SIRT3 over-expression has been shown to lower mitochondrial membrane potential $(\Delta \Psi \mathrm{m})$ and ROS levels in different non-neuronal cell types (Shi et al., 2005; Bell and Guarente, 2011; Chen et al., 2011; Shulyakova et al., 2014; Liu et al., 2015; Pillai et al., 2016; Ren et al., 2016). While the exact mechanism of how SIRT3 decreases $\Delta \Psi \mathrm{m}$ and ROS levels remain to be fully elucidated, a potential mechanism has been suggested for brown adipose tissue (Shi et al., 2005). In these cells, there is a SIRT3-dependent up-regulation of the transcriptional regulator peroxisome proliferator-activated receptor gamma coactivator 1-alpha (PGC1- $\alpha$ ) expression, a transcriptional co-activator that regulates the expression of numerous genes involved in mitochondrial function and detoxification pathways (Onyango et al., 2010). PGC1- $\alpha$ facilitates an increase in the expression levels of mitochondrial uncoupling protein 1 (UCP1), which in turn decreases $\Delta \Psi \mathrm{m}$ by enhancing proton flux back across the inner mitochondrial membrane into the matrix (Klingenspor, 2003). The lower $\Delta \Psi \mathrm{m}$ decreases the driving force for electron leak during oxidative phosphorylation, and thereby contributes to the decrease in ROS genesis (Sullivan et al., 2004). It is unlikely that this direct mechanism would apply to the brain, however, as UCP1 expression is low or undetectable in the CNS under normal conditions (Andrews et al., 2005) and therefore the function of SIRT3 in brain does not likely involve UCP1. It is worth noting though that other relatives of UCP1 are expressed in the brain (UCP2', -4 and -5$)^{\prime}$, and their elevated expression and activity have also been correlated with neuroprotection (Andrews et al., 2005). It is therefore possible that these UCP family members could potentially substitute for UCP1 in neurons as targets for SIRT3. To date, though, this remains speculative, as direct SIRT3 regulation of any UCP family member in neurons remains undetermined.

\section{SIRT3 Influences the Expression of Nuclear Genes Encoding Anti-Oxidative and Mitochondrial Factors}

In addition to its direct activation of antioxidant target proteins by deacetylation, SIRT3 has also been shown to indirectly 
regulate the expression of specific antioxidant enzymes. Jacobs et al. (2008) showed that the transcription factor forkhead box O3 (FOXO3a) is a substrate of SIRT3 in mitochondria, which upon deacetylation translocates into the nucleus to stimulate the transcription of specific anti-oxidative genes. As stated above, SIRT3 directly or indirectly also influences the expression of PGC-1 $\alpha$. Although the mechanism of SIRT3 regulation of PCG- $1 \alpha$ is not fully understood, it has been speculated that SIRT3 indirectly increases the phosphorylation of the transcription factor, cAMP response element-binding protein (CREB; Shi et al., 2005; Kong et al., 2010), which then facilitates PGC-1 $\alpha$ gene expression. Thus, SIRT3 regulates mitochondrial proteins at both their expression and functional levels by indirectly regulating their gene-expression in the nucleus, and their catalytic/functional state directly in mitochondria through post-translational deacetylation. These results further illustrate how SIRT3 could be viewed as a key rheostat for maintaining mitochondrial homeostasis.

\section{SIRT3 Influence on Mitochondrial Permeability Transition Pore Activation}

In pathological conditions and following different neural injuries such as stroke or trauma, the formation and opening of the mitochondrial permeability transition pore ( $\mathrm{mPTP})$ is a hallmark of end-stage mitochondrial dysfunction that allows for the release of pro-apoptotic factors from mitochondria that activate cell death pathways (Halestrap and Pasdois, 2009; Sims and Muyderman, 2010). Inhibiting mPTP opening has been proposed as a neuroprotective strategy for a number of neurodegenerative diseases (Rao et al., 2014), and one proposed mechanism for SIRT3-mediated cytoprotection involves it inhibiting the opening of the MPTP (Hafner et al., 2010). In cardiac myocytes, Hafner et al. (2010) showed that SIRT3 deacetylase activity inhibited the formation of the MPTP by decreasing the binding of cyclophilin D (CypD) to adenine nucleotide translocase (ANT). The SIRT3-mediated regulation of CypD is evident in brain, as CypD hyperacetylation is observed in hippocampal tissues and cortical neurons isolated from SIRT3-KO mice (Cheng et al., 2016). These results suggest that SIRT3 activity in brain negatively influences mPTP opening by maintaining CypD in a deacetylated state. However, there may be context-specificity or limits to this action of SIRT3, as Novgorodov et al. (2016) found no difference in $\mathrm{Ca}^{2+}$-induced $\mathrm{mPTP}$ opening in mitochondria isolated from cerebral tissues that were harvested from ischemiachallenged SIRT3-KO mice and wild-type mice. However, the requisite role of acetyl-CypD on $\mathrm{mPTP}$ opening may also be context-dependent, as mPTP opening occurred without any CypD acetylation state changes in peroxide-treated H9c2 rat embryonic cardioblasts (Barreto-Torres et al., 2015), and ParodiRullam et al. (2017) found no difference in mPTP opening and CypD acetylation in hearts between WT and SIRT3-KO mice following ischemia-reperfusion injury. Further, CypD itself may influence mitochondrial acetylation levels, as mice lacking CypD show hyperacetylation of mitochondrial proteins without any change in SIRT3 expression or activity levels (Nguyen T. T. M. et al., 2013). These studies suggest that the cause-effect between
CypD acetylation by SIRT3 and the opening of MPTP is complicated and may be context-dependent. Therefore, future studies are necessary to elucidate the role of SIRT3 on the opening of mPTP under normal physiological and different pathophysiological conditions.

\section{SIRT3 Influence on Mitochondrial Biogenesis and Mitochondrial Dynamics}

SIRT3 has also been implicated in mitochondrial biogenesis regulation (Shi et al., 2005; Kong et al., 2010; Giralt et al., 2011; Dai et al., 2014; Zhao et al., 2016), mitochondrial fusion/fission dynamics (Song et al., 2013; Samant et al., 2014; Morigi et al., 2015), and related aspects of maintaining mitochondrial integrity. Mitochondrial biogenesis is a process through which new mitochondria are generated. This phenomenon is especially important for neural development and may also serve as a protective mechanism during neurodegenerative disease (Onyango et al., 2010). Indeed, studies show that SIRT3 expression is required for proper mitochondrial biogenesis in both mouse $\mathrm{C}_{2} \mathrm{C}_{12}$ myoblasta (Kong et al., 2010) and human oocytes (Zhao et al., 2016). Mitochondrial dynamics also play an important role in the brain, as mitochondria continuously undergo dynamic changes in their size, shape, number, and distribution to appropriately respond to fluctuating metabolic demands (Milone and Benarroch, 2012). Fission results in mitochondrial division, while fusion allows distinct mitochondria to exchange intra-mitochondrial metabolic products and mtDNA. This normal fission/fusion balance can be altered by various external factors, such as ROS elevation (Wu et al., 2011), reduced ATP levels (Mishra and Chan, 2016) and mitochondrial $\mathrm{Ca}^{2+}$ influx (Chen and Chan, 2009; Kaddour-Djebbar et al., 2010). Samant et al. (2014) reported that SIRT3 preserves mitochondrial networking in cardiomyocytes by deacetylating and activating the GTPase activity of optic atrophy 1 (OPA1), which is an integral protein involved in promoting mitochondrial fusion. In addition, Morigi et al. (2015) reported that SIRT3 over-expression prevented the mitochondrial recruitment of the fission-promoting protein dynamin-related protein-dependent (DRP1) and also increased OPA1 expression in cultured human renal proximal tubular epithelial cells in response to fission-inducing cisplatin challenge. As mitochondrial fragmentation is evident in many neurodegenerative conditions such as Alzheimer's disease (AD; Wang et al., 2009), Parkinson's disease (Van Laar and Berman, 2009), amyotrophic lateral sclerosis (ALS; Jiang et al., 2015), and at early stages following reversible ischemic stroke (Barsoum et al., 2006), it will be of considerable interest to determine whether SIRT3 can be harnessed to preserve mitochondrial integrity in these conditions and circumstances.

\section{SIRT3 Regulates Energy Source Utilization by Mitochondria}

SIRT3-mediated deacetylation and activation of several factors involved in mitochondrial energy production have been demonstrated. These include acetyl coenzyme A synthetase type 2 (AceCS2), glutamate dehydrogenase (GDH), IDH2 type 2, 
and specific components of the electron transport chain (ETC; Hallows et al., 2006; Schlicker et al., 2008; Someya et al., 2010; Yu et al., 2012). AceCS2 catalyzes formation of acetyl-CoA from free acetate and coenzyme A (CoA), which can then be used via the TCA cycle for ATP synthesis (Fujino et al., 2001). The function of AceCS2 is especially important during periods of diminished glucose availability, as in such times of need the liver can release significant amounts of stored acetate for use by extra-hepatic tissues that include the brain (Yamashita et al., 2001). SIRT3 activation of GDH enhances the production of TCA cycle intermediates $\alpha$-ketoglutarate and $\mathrm{NADH}$, which enhances the pool of electron donating substrates for oxidative phosphorylation (Schlicker et al., 2008). In this regard, SIRT3 has also been shown to deacetylate and regulate the activity of complex I in general (Kim et al., 2010), and specific mitochondrial respiratory chain complex components such as NDUFA9 of Complex I (Ahn et al., 2008); Succinate Dehydrogenase of complex II (Finley et al., 2011) and ATP synthase subunits $\alpha$ and $\beta$ (Law et al., 2009; Wu et al., 2013; Rahman et al., 2014). In the absence of SIRT3, the activities complex I, complex II, and complex V were each shown to be decreased relative to control (Ahn et al., 2008; Law et al., 2009; Finley et al., 2011; Rahman et al., 2014; Novgorodov et al., 2016), and the activity of complexes III and IV were also found to be decreased in liver extracts from SIRT3-KO mice maintained on a high fat diet (Kendrick et al., 2011). Consistently, decreased ATP levels were reported in several different organs of SIRT3-KO mice (Ahn et al., 2008). It should be noted, however, that these observations are not absolute, as Fernandez-Marcos et al. (2012) reported no changes in ATP levels in SIRT3-KO mouse muscle or liver, and Novgorodov et al. (2016) reported that complex I activity but not complex II or complex IV activity was reduced under basal conditions in SIRT3-KO mouse cerebral cortex. Interestingly, a decrease in basal mitochondrial oxygen consumption rate has been observed in primary cultured mouse dopaminergic neurons overexpressing SIRT3 (Gleave et al., 2017), suggesting that SIRT3 over-expression increases mitochondrial efficiency by stabilizing respiration at the electron transport level. While these results support SIRT3 as a factor regulating overall cellular energy production at different levels, additional studies are needed to better define how changes in metabolic state affect the activity of SIRT3, and how SIRT3 influences mitochondrial respiratory activity in highly erobic tissues such as brain.

\section{SIRT3 Influences the Production of Energy Substrates}

Brain metabolic demand is regulated by a complex interplay of behavioral state, energy substrate supply and availability, and hormonal signaling between periphery and tissues within the central nervous system (Lenard and Berthoud, 2008). The energy source primarily used by the brain is glucose, and tight regulation of its metabolism is critical for normal brain physiology, structure integrity, and neuronal viability (Mergenthaler et al., 2013; Bauernfeind et al., 2014). However, when glucose availability becomes limited, the brain is able to utilize alternative sources from other sources in the body for energy production (White and Venkatesh, 2011; Prins, 2012; Glenn et al., 2015; Proia et al., 2016). Glycogen reserves can be tapped to maintain bloodglucose levels acutely, and meet the high-metabolic demands of the brain for a short period of time (Waitt et al., 2017). Once these glycogen reserves have been depleted, though, another alternative energy source must serve as substrates. During such conditions, fatty acid reserves from adipose tissues can be catabolized, and used for fatty acid $\beta$-oxidation in the liver to generate acetyl-CoA, which is then converted by ketogenesis into ketone bodies (White and Venkatesh, 2011). These ketones are then carried to metabolically active tissues, such as the brain, where they are converted back to acetyl-CoA to serve as an energy source. Studies have suggested SIRT3 plays a vital role in all the major steps of this hepatic "glucose-sparing energy pathway", as SIRT3 deacetylates and activates two key enzymes involved in fatty acid $\beta$-oxidation pathway initiation: long-chain acyl CoA dehydrogenase (LCAD; Hirschey et al., 2010) and very long-chain acyl-CoA dehydrogenase (VLCAD Hallows et al., 2011; Zhang et al., 2015). These interactions have been demonstrated in liver, but it is worth noting that both LCAD and VLCAD are also expressed in brain, and could therefore also be subject to SIRT3 regulation in the CNS. SIRT3 has also been implicated in activating L-3-hydroxyacyl-CoA dehydrogenase, short/branched-chain acyl-CoA dehydrogenase and 3-ketoacylCoA thiolase (Hallows et al., 2011), which are each also involved in $\beta$-oxidation processes. Moreover, SIRT3 has also been shown to regulate the production of ketone bodies by targeting 3-hydroxy-3-methylglutaryl-CoA synthase (HMGCS2), which is the limiting enzyme in the production of ketone bodies from acetyl-CoA and acetoacetyl-CoA (Shimazu et al., 2010). Intriguingly, SIRT3 not only promotes the synthesis of ketones, it also shifts the hepatic mitochondrial metabolism to meet the higher energy demand that is necessary for ketogenesis. This is accomplished by deacetylating and activating the mitochondrial transcription factor leucine-rich protein 130 (LRP130), who in turn stimulates the transcription of genes in the mitochondrial genome encoding factors required for oxidative phosphorylation cascades (Liu et al., 2011). Whether this occurs similarly in brain as demonstrated in liver remains to be determined.

In addition to ketone bodies, lactate is another alternative source of energy used by the brain under certain circumstances. Lactate can be transported from the periphery across the BBB by monocarboxylate transporters 1 (MCT1) using facilitated diffusion, and within the brain astrocytes can also serve a source of lactate for neurons (reviewed in Steinman et al., 2016). Neurons express MCT2, which transports lactate intracellularly where lactate dehydrogenase $(\mathrm{LDH})$ converts it into pyruvate, which is then converted to acetyl-CoA by pyruvate dehydrogenase (PDH; Pellerin et al., 2005). Although a direct link has not yet been established in neural tissues, SIRT3 has been shown to deacetylate both LDH (Cui et al., 2015) and PDH in a number of cancer cells (293T, HCT116, HeLa, T47D, MMT and MCF7; Ozden et al., 2014) and mouse skeletal muscle (Jing et al., 2013). These data therefore provide another potential means through which SIRT3 could facilitate the use of alternative energy 


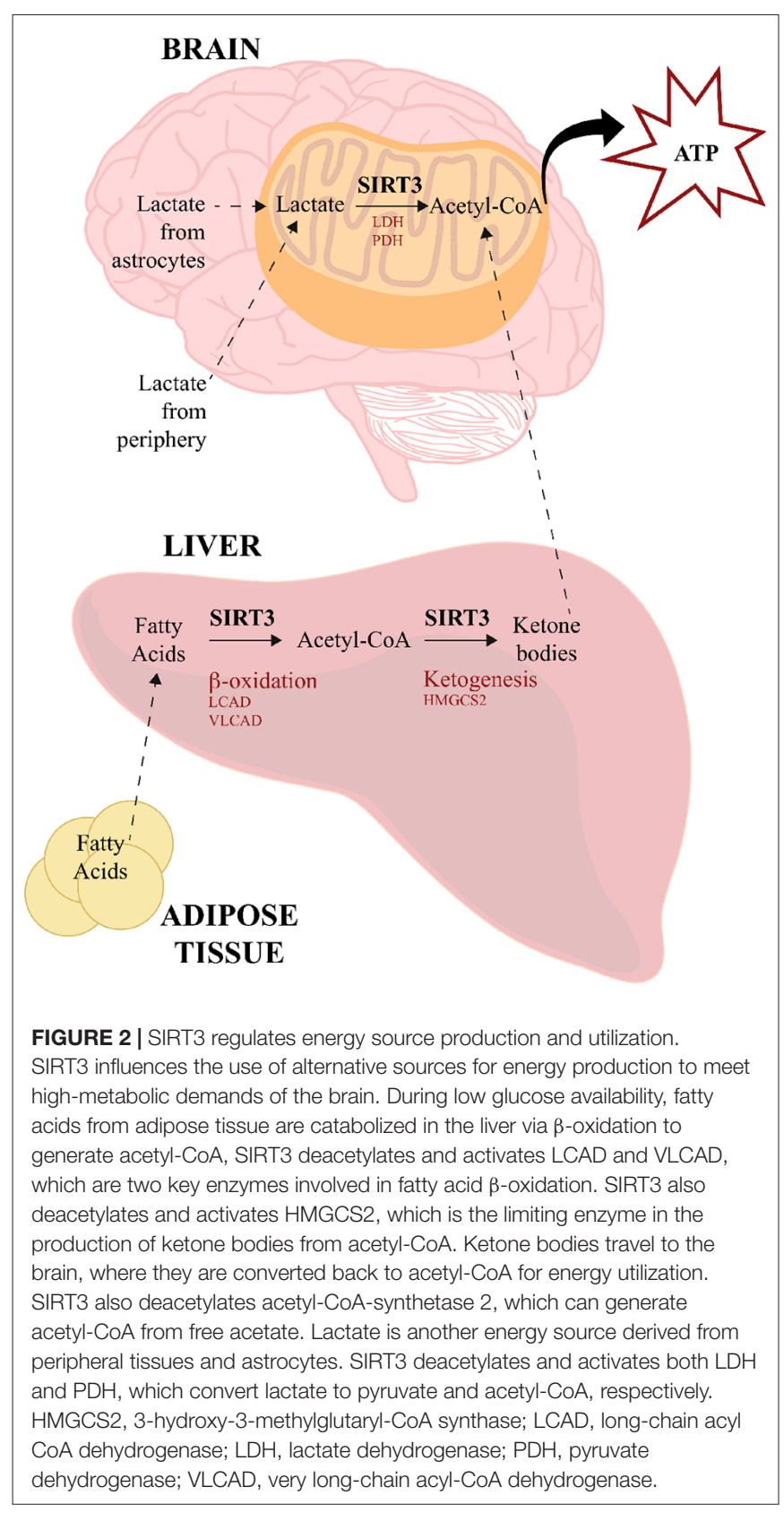

sources in the brain during times of need. The visual overview of how SIRT3 regulates energy source production and utilization is illustrated in Figure 2.

\section{THE POTENTIAL ROLE OF SIRT3 IN NEURODEGENERATIVE DISEASES}

Although neurodegenerative diseases manifest with different clinical outcomes, their pathophysiology often converges at the level of mitochondrial dysfunction (Martin, 2012; Morris and Berk, 2015). While there is considerable debate of whether mitochondrial dysfunction is causal to, or consequential of, the progression of these CNS there is general agreement that improving mitochondrial homeostasis and functional capacity could improve outcomes for many degenerative conditions. Thus, elucidating the role of SIRT3 in the progression of these conditions, or for its potential to be targeted to alter disease course, are emerging areas of interest (see Supplementary Table S1).

\section{SIRT3 in Alzheimer's Disease}

Growing evidence has demonstrated mitochondrial dysfunction is a critical factor contributing to the initiation and progression of AD (Grimm et al., 2016), and recent data suggest SIRT3 may play a role in this process. Expression analysis has revealed changes in SIRT3 expression levels in post-mortem samples from $\mathrm{AD}$ patients and in experimental mouse models. For example, Weir et al. (2012) reported increased SIRT3 mRNA levels in human post-mortem midfrontal and temporal neocortical tissues from AD patients (Weir et al., 2012), while in contrast, Yin et al. (2018) reported decreased SIRT3 mRNA and protein levels in human $\mathrm{AD}$ post-mortem cortical regions (Supplementary Table S1). The reason for the discrepancy in SIRT3 expression outcome is unclear, but the results do suggest an AD-related SIRT3 response does occur during the course of the disease.

In mouse models, SIRT3 expression responses have also been identified, but like in $\mathrm{AD}$ patients somewhat different outcomes have been observed in different models. Sirt3 mRNA levels were initially elevated in hippocampal tissues of early symptomatic 6 month-old AD PDAPP mice (which overexpress a human amyloid beta (A $\beta$ ) precursor protein (APP) carrying the V717F mutation), but this increase in SIRT3 expression returns to normal levels by 26 months of age (Weir et al., 2012). Both SIRT3 mRNA and protein levels were significantly diminished in APP/PS1 double transgenic mice at 12 months of age (Yang et al., 2015), and decreased SIRT3 expression was similarly found in PSEN1/APP/TAUP301L mice at 24-months of age (Han et al., 2014). The initial up-regulation of SIRT3 may represent an endogenous neuroprotective response early in disease pathogenesis that is then lost as the disease further progresses. Furthermore, over-expression of SIRT3 prevented $A \beta-42$ induced tau accumulation in cultured cortical neurons derived from mice expressing a human tau transgene, while knockdown of SIRT3 in the same cultured neurons caused an elevation of tau accumulation (Yin et al., 2018). This observation is consistent with that of another recent study which showed treating hamster ovary PS70 cells expressing mutant APP with the SIRT3 activator Honokiol attenuated ROS levels, increased mitochondrial membrane potential, elevated the levels of p-CREB and PGC1 $\alpha$ and importantly decreased intracellular levels of $\mathrm{A} \beta$ (Ramesh et al., 2018). These results collectively suggest SIRT3 activity may attenuate the over-production and accumulation of tau. While encouraging, further studies will be required to define the contribution and potential translational value of SIRT3 in AD pathogenesis.

\section{SIRT3 in Parkinson's Disease}

Two agents widely used to induce experimental Parkinsonism disease (PD) in model systems are 1-methyl-4-phenyl- 
1,2,3,6-tetrahydropyridine (MPTP) and rotenone; both of which are believed to cause PD-like degeneration through their induction of oxidative stress levels and impairment of mitochondrial function. In cultured SHSY-5Y cells, Cui et al. (2017) found that SIRT3 over-expression prevented the ATP loss induced by $\mathrm{MPP}^{+}$(a metabolite of MPTP) treatment, and SIRT3 over-expression also diminished apoptosis, $\alpha$-synuclein accumulation, and cell death following rotenone treatment of the same cells (Zhang et al., 2016). Consistently, SHSY-5Y cells lacking SIRT3 showed an increase in apoptosis, $\alpha$-synuclein accumulation, and cell death following the same rotenone treatment (Zhang et al., 2016). In line with the in vitro studies, SIRT3-KO mice showed exacerbated degeneration of dopaminergic neurons compared to wild-type mice following MPTP treatment (Liu et al., 2015), and viral-mediated over-expression of SIRT3 protected dopaminergic neurons from $\alpha$-synucleininduced degeneration (Gleave et al., 2017). Shi et al. (2017) also observed an increase in oxidative stress and degeneration of SNc dopaminergic neurons from SIRT3-KO mice, which correlated with increased lysine-68 acetylation of MnSOD (MnSODK68). Importantly, SNc dopaminergic neuron degeneration was prevented by either reintroduction of functional SIRT3, or by introduction of a constitutively active deacetylation-mimetic form of MnSODK68 (Shi et al., 2017). Collectively, these studies suggest that the endogenous functions of SIRT3 may indeed play a protective role in PD pathogenesis, and suggest SIRT3 may represent a therapeutic target for PD translational development, particularly since hyperacetylation of MnSODK68 has been observed in post-mortem midbrain tissues from PD patients (Shi et al., 2017).

\section{SIRT3 in Amyotrophic Lateral Sclerosis}

Although there have been few investigations of SIRT3 to date in ALS models, the available data suggest the actions of SIRT3 could influence ALS pathogenesis. As discussed above, (Song et al., 2013) showed the mitochondrial fragmentation and activation of apoptotic cascades in cultured motor neurons derived from SOD1 ${ }^{\mathrm{G} 93 \mathrm{~A}}$ mutant mice could be prevented by the ectopic over-expression of SIRT3. Similarly, Harlan et al. (2016) found that SIRT3 over-expression in SOD ${ }^{\mathrm{G} 93 \mathrm{~A}}$ mutant mouse astrocytes rescued the degeneration of co-cultured SOD1 ${ }^{\mathrm{G} 93 \mathrm{~A}}$ mutant mouse motor neurons. Further studies are clearly required, but these results suggest that enhancing SIRT3 activity may provide a protective role in at least for some forms of ALS.

\section{SIRT3 in Huntington's Disease}

Although the mechanism in neuronal degeneration is not fully understood in Huntington's disease (HD), mitochondrial involvement has been speculated (Chen, 2011). To date, only one study has examined SIRT3 in the context of HD progression (Fu et al., 2012). Fu et al. (2012) showed that SIRT3 protein levels and deacetylase activity are diminished in a human striatal precursor cell line expressing mutant $\mathrm{Htt}$, and the protective effects observed in these cells following treatment with the stilbenic compound $n s$ $(-)-\varepsilon$-Viniferin (viniferin) were lost when endogenous SIRT3 was knocked down. Although further studies are required, this initial study also suggest SIRT3 up-regulation or enhancement of enzymatic activity could slow the progression of HD.

\section{The Role of SIRT3 Can Be Cytoprotective or Cytodamaging in Models of Stroke}

To date, several studies have examined the role of SIRT3 in different in vitro and in vivo models of stroke. Kim et al. (2011) reported that the over-expression of SIRT3 in cortical neurons protected against NMDA-excitotoxicity; Dai et al. (2017) demonstrated lentiviral over-expression of SIRT3 protected cultured cortical neurons from an oxygen/glucose deprivation (OGD) challenge; Shulyakova et al. (2014) showed SIRT3 over-expression protected neuronally differentiated PC12 cells from OGD and from apoptotic degeneration induced by trophic withdrawal; Magnifico et al. (2013) found that transient over-expression of full-length mouse SIRT3 protected cultured mouse cerebellar granule neurons from axonal degeneration induced by $\mathrm{KCl}$ depletion; and Yang et al. (2017) found that administration of the SIRT3 activator adjudin attenuated glial scar formation and improved functional recovery in mice following transient middle cerebral artery occlusion (MCAO), but that these effects were lost in SIRT3-KO mice. Collectively, these studies support the hypothesis that enhancing SIRT3 prevalence and/or activity can provide protection against a spectrum of insult-related challenges.

However, not all experimental data indicate that the over-expression or increased activation of SIRT3 is beneficial. In fact, quite contrasting outcomes have been observed in some experimental systems. For example, cerebellar granule neurons overexpressing SIRT3 displayed enhanced neuronal death in response to low potassium ion treatment (Pfister et al., 2008), and SIRT3-KO mice displayed smaller brain infarct volumes than wild type mice following reversible MCAO (Novgorodov et al., 2016), and Verma et al. (2018) also recently reported ischemia/reperfusion damage to be reduced in SIRT3-KO mice. In the Novgorodov et al., 2016 study, Sirt3 ablation was found to lessen the activation of ceramide synthases 1,2 and 6 following reperfusion, which was hypothesized to help preserve the overall function of the challenged mitochondria by decreasing the generation and accumulation of toxic mitochondrial ceramides (reviewed in Novgorodov and Gudz, 2011). The Verma et al. (2018) study employed a similar model, but speculated the observed protection may relate to a compensatory up-regulation of the SIRT1 sirtuin in the brains of SIRT3 KO mice. These results do not necessarily contradict the anti-oxidant or anti-apoptotic capacity of SIRT3, as the severity or type of stroke challenge could differentially activate ROS-dependent or ceramide-dependent cell death cascades (Novgorodov and Gudz, 2011; Mencarelli and Martinez-Martinez, 2013), but they do highlight the possibility that SIRT3 may differentially regulate stroke-related outcomes in tissue- 
or context-dependent manners (Dittenhafer-Reed et al., 2015).

\section{REGULATION OF SIRT3 EXPRESSION LEVELS AND FUNCTION}

Converging data now suggest that augmenting SIRT3 activity may be beneficial in progressive neurodegenerative diseases such as $\mathrm{AD}$ and $\mathrm{PD}$, and that modulating SIRT3 activity may also provide protection in different types of stroke depending on context. This section will review current knowledge of endogenous and pharmacological regulators of SIRT3 expression and catalytic activity.

\section{The Role of Diet and Exercise in Regulating SIRT3 Expression Levels}

While the molecular mechanisms remain to be fully elucidated, both diet and exercise have been shown to influence SIRT3 expression levels. Mice maintained on caloric restriction (CR) diets show increased SIRT3 mRNA and protein levels in a host of tissues, which include brown adipose tissue (Shi et al., 2005), skeletal muscle (Palacios et al., 2009; Tauriainen et al., 2011), liver (Tauriainen et al., 2011), adipose tissue (Tauriainen et al., 2011) and brain (Amigo et al., 2017). However, these expression outcomes depend on the degree of CR, as studies also found $48 \mathrm{~h}$ of complete fasting decreased SIRT3 mRNA in human muscle tissue (Edgett et al., 2016). Exercise was also found to increase SIRT3 expression in both rat and mouse striated muscle and brain (Ferrer et al., 2009; Palacios et al., 2009; Hokari et al., 2010; Brandauer et al., 2015; Cheng et al., 2016). Some data have implicated the SIRT3 up-regulation stemming from $C R$ and exercise to arise at least in in part through the AMP-activated protein kinase (AMP-kinase) pathway (Brandauer et al., 2015), as following nutrient restriction and exercise there is an increase in AMP-kinase activity due to elevated AMP/ATP ratios (Richter and Ruderman, 2009; Cantó and Auwerx, 2011). While AMP-kinase has several targets depending on context and cell type, one intriguing target of AMP-kinase is the transcriptional regulator CREB, which is known to activate PGC-1 $\alpha$ expression under several conditions (Thomson et al., 2008; Palacios et al., 2009). There may also be convergence on CREB as a regulating factor for SIRT3 expression, as Cheng et al. (2016) found that increases in SIRT3 expression in mouse brain following exercise were abolished by treatment with the NMDA receptor antagonist MK801. These results thereby implicate glutamatergic systems, which are well established to influence activity-dependent CREB signaling (Schurov et al., 1999; Lonze and Ginty, 2002) in exercise-induced SIRT3 expression regulation. This would also be consistent with the elevation of SIRT3 protein levels that were observed in cultured cortical mouse neurons following acute treatment with NMDA in vitro (Kim et al., 2011). These collective results therefore suggest both activity-dependent and AMP-kinase-related mechanism could contribute to exerciserelated SIRT3 up-regulation in brain, and this up-regulation of SIRT3 underlie some of the general benefits associated with exercise (Radak et al., 2016).
Regulation of SIRT3 Deacetylase Activity

Accumulating evidence indicates SIRT3 enzymatic activity fluctuates in accordance with the metabolic state of the cell (Shi et al., 2005; Ferrer et al., 2009; Palacios et al., 2009; Hokari et al., 2010; Li et al., 2011; Tauriainen et al., 2011; Dai et al., 2014; Brandauer et al., 2015; Cheng et al., 2016; Amigo et al., 2017). Because SIRT3 uses $\mathrm{NAD}^{+}$as a cofactor for deacetylation reactions, the ratio of $\mathrm{NAD}^{+} / \mathrm{NADH}$ within the mitochondria can influence its overall deacetylase activity. $\mathrm{NAD}^{+} / \mathrm{NADH}$ ratios can be dynamic within cells, particularly in metabolically demanding cells such as neurons (Cantó et al., 2015). As the deacetylation reaction catalyzed by SIRT3 results in the conversion of $\mathrm{NAD}^{+}$into nicotinamide and acetylADP-ribose, high SIRT3 activity could potentially deplete $\mathrm{NAD}^{+}$within mitochondrial microdomains, and thereby limit its own catalytic activity by removing its required co-factor. Moreover, nicotinamide itself is an endogenous inhibitor of the SIRT3 deacetylation reaction (Guan et al., 2014), which suggests the generation of nicotinamide by SIRT3 could feed-back to attenuate its own activity. Finally, it is also intriguing that $\mathrm{NAD}^{+} / \mathrm{NADH}$ ratios tend to increase during metabolic states associated with CR and exercise (Hipkiss, 2008)-which are recognized for their neuroprotective and/or lifespan extension potential (reviewed in Deslandes, 2013; Michan, 2014). In this regard, CR diets increase SIRT3 deacetylase activity in a number of tissues, which include brain (Someya et al., 2010; Amigo et al., 2017), liver (Someya et al., 2010) and inner ear (Someya et al., 2010) and many of the ascribed benefits of both CR and exercise are lost when SIRT3 activity is abrogated (Palacios et al., 2009; Someya et al., 2010; Cheng et al., 2016). These results therefore provide evidence for an intricate link between $\mathrm{CR}$, exercise, increased $\mathrm{NAD}^{+} / \mathrm{NADH}$ ratios, SIRT3 enzymatic activity, and cytoprotective effects associated with exercise and/or CR.

\section{Genetic Regulation of SIRT3 Expression Levels}

Although still a work in progress, several factors that influence the gene and protein expression levels of SIRT3 are beginning to be delineated in specific model systems. At the genomic level, PGC-1 $\alpha$ appears to be a key positive regulator of SIRT3 expression (Kong et al., 2010). As such, conditions within cells that induce PGC- $1 \alpha$ activity may also induce SIRT3 gene expression. This may not be surprising, as PGC$1 \alpha$ is well known to activate the expression of a host of mitochondrial and anti-oxidative defense factors (Onyango et al., 2010). However, the mechanisms through which PGC-1 $\alpha$ induces SIRT3 expression are complex and may display cell-type specificity. For example, PGC- $1 \alpha$ does not appear to bind to the SIRT3 promoter region directly; rather it induces the expression of other genes whose products partner with PGC- $1 \alpha$ and bind to a region of the SIRT3 promoter as heterodimers. Two such target genes activated by PGC-1 $\alpha$ are estrogen-related receptor $\alpha$ (ERR $\alpha$; Kong et al., 2010), and nuclear respiratory factor 2 (NRF2; Mootha et al., 2004). Both ERR $\alpha$ and NRF2 bind to distinct sites located proximal to the SIRT3 transcriptional start site, and are suggested to be co-dependent on PGC-1 $\alpha$ for this 
binding activity (Kong et al., 2010; Satterstrom et al., 2016). In addition to regulating SIRT3 expression, ERR $\alpha$ and NRF2 also partner with PGC- $1 \alpha$ to up-regulate the expression of other nuclear genes encoding mitochondrial factors (Mootha et al., 2004; Eichner and Giguère, 2011). Intriguingly, the activities of at least some of the proteins derived from these genes are also regulated by SIRT3 deacetylase activity once they reach the mitochondria.

\section{Therapeutic Approaches to Regulate SIRT3 Expression Levels and Function}

The activity of SIRT3 can be upregulated by a number of pharmacological (or chemical) compounds. The first substance suggested to increase the activity of specific sirtuin family members was resveratrol (3,4',5-trihydroxystilbene; RSV; Howitz et al., 2003), a polyphenol found in red wine speculated to increase anti-oxidative defense when consumed in moderate levels (Sun et al., 2010; Singh et al., 2013). Although RSV has been reported to left-shift the Michaelis constant of SIRT1 for substrate (Howitz et al., 2003), its actions on SIRT3 remain more controversial. RSV treatment has been reported to elevate SIRT3 levels in mouse cardiac fibroblasts (Chen et al., 2015) and increase SIRT3 enzymatic activity in HepG2 cells (Desquiret-Dumas et al., 2013). However, RSV treatment was also reported to down-regulate sirt3 mRNA levels in zebrafish liver (Schirmer et al., 2012), and inhibits human SIRT3 (Gertz et al., 2012). Other RSV-like compounds which are touted to be more stable and potent than resveratrol, such as piceatannol and 4'-bromo-resveratrol (5-(2-(4-hydroxyphenyl)vinyl)-1,3-benzenediol; Gertz et al., 2012; Nguyen G. T. et al., 2013) have inhibitory effects on SIRT3 activity. As discussed above, it is important to note that the activity of SIRT3 is dependent upon $\mathrm{NAD}^{+}$levels, and there is evidence that $\mathrm{NAD}^{+}$levels can be indirectly affected following RSV treatment. For example, Desquiret-Dumas et al. (2013) showed that RSV directly enhances complex $\mathrm{I}$ activity, and thereby increases $\mathrm{NAD}^{+} / \mathrm{NADH}$ ratios. The positive regulation of SIRT3 through this resveratrol-NAD ${ }^{+}$ axis is in line with observations that supplementation with the $\mathrm{NAD}^{+}$precursor nicotinamide riboside similarly enhance SIRT3 activity in mammalian cells and mouse tissues (Brown et al., 2014). Thus, it is possible some of the effects associated with resveratrol may stem from indirect effects on $\mathrm{NAD}^{+}$ levels.

Other molecules that are structurally distinct from RSV have also been demonstrated to regulate SIRT3 enzymatic activity. Aside from the global sirtuin inhibitor nicotamide (Bitterman et al., 2002), which as discussed above inhibits SIRT3 by competing with $\mathrm{NAD}^{+}$at the SIRT3 cofactor binding site (Guan et al., 2014), some examples of SIRT3 inhibitory modulators are SRT1720 (Jin et al., 2009), 5-amino-2-phenylbenzoxazole (Salo et al., 2013), LC-0296 (Alhazzazi et al., 2016) and SDX-437 (Patel et al., 2015). Of these, SDX-437 and LC-0296 showed the highest apparent selectivity for SIRT3 over SIRT1 (Patel et al., 2015; Alhazzazi et al., 2016). At present, though, these compounds remain largely untested for their ability to modulate SIRT3 in vivo, and specifically within the CNS.
With respect to SIRT3 activators, several have been reported, such as oroxylin A (OA; Zhao et al., 2015), viniferin (Fu et al., 2012), HKL [2-(4-hydroxy-3-prop-2-enyl-phenyl)-4-prop2-enyl-phenol] (Pillai et al., 2015), nitrite (Lai et al., 2016), adjudin (Yang et al., 2017), 7-hydroxy-3-(4'-methoxyphenyl) coumarin (C12; Lu et al., 2017), although similar to what has been observed for inhibitors, the specificity for SIRT3 in vivo in the brain remains untested. This is an emerging area, however, with new molecules and analogs being developed rapidly.

\section{CONCLUSIONS AND SUMMARY}

Taken together, current research sheds light on the widespread role that SIRT3 activity may play in regulating normal brain function, and how its activities could influence the pathogenic course of stroke and different neurodegenerative diseases. Mitochondrial function is cornerstone to maintaining the homeostatic balance of cells, and particularly those with high energetic demands such as neurons.

SIRT3 has now been shown to post-translationally deacetylate and regulate the activity of a number of mitochondrial proteins whose functions are salient for oxidative phosphorylation and for maintaining anti-oxidative defense, and recent work shows SIRT3 is prominently expressed in central nervous system tissue. Given this, it is perhaps surprising that SIRT3 KO mice display only modest physiological or behavioral alterations under baseline conditions (Lombard et al., 2007; Liu et al., 2015). Whether or not exacerbations in behavioral impairments will be more evident under stressful conditions or as a consequence of aging remains largely to be determined, but the targets of SIRT3 that have been identified makes this a plausible possibility. While, SIRT3 deficiency does cause a hyperacetylation of the mitochondrial proteome (Lombard et al., 2007; Cheng et al., 2016), the consequences of SIRT3 over-expression remain less clear, as examinations of the global acetylation state of mitochondria remains to be assessed. Therefore, it is unclear whether the consequences observed in SIRT3 over-expression models stem from global mitochondrial proteome hypoacetylation, or from the regulation of specific SIRT3 targets. There justifiably remains much interest in this specific mitochondrial sirtuin, and while much information has been gleaned, many important questions - particularly within the context of the nervous system-remain to be answered.

\section{AUTHOR CONTRIBUTIONS}

ES-D conducted literature review, compiled data and wrote the manuscript. RS assembled Tables and proofed the manuscript. JE oversaw project, edited and compiled the final manuscript.

\section{FUNDING}

ES-D is the recipient of an Ontario Graduate Scholarship, an Ontario Heart and Stroke Foundation Scholarship, a Peterborough K.M. Hunter Graduate Scholarship and a Norman Stuart Robertson Fellowship. RS received Undergraduate 
Research Opportunity Program Scholarship. This work was supported by operating grant NA-7041 from the Heart and Stroke Foundation of Canada to JE.

\section{ACKNOWLEDGMENTS}

We would like to thank members of the Eubanks laboratory and Dr. Linda Mills for helpful discussions relating to this

\section{REFERENCES}

Ahn, B. H., Kim, H. S., Song, S., Lee, I. H., Liu, J., Vassilopoulos, A., et al. (2008). A role for the mitochondrial deacetylase Sirt3 in regulating energy homeostasis. Proc. Natl. Acad. Sci. U S A 105, 14447-14452. doi: 10.1073/pnas. 0803790105

Ahuja, N., Schwer, B., Carobbio, S., Waltregny, D., North, B. J., Castronovo, V., et al. (2007). Regulation of insulin secretion by SIRT4, a mitochondrial ADP-ribosyltransferase. J. Biol. Chem. 282, 33583-33592. doi: 10.1074/jbc. m705488200

Alhazzazi, T. Y., Kamarajan, P., Xu, Y., Ai, T., Chen, L., Verdin, E., et al. (2016). A novel sirtuin-3 inhibitor, LC-0296, inhibits cell survival and proliferation and promotes apoptosis of head and neck cancer cells. Anticancer Res. 36, 49-60.

Amigo, I., Menezes-Filho, S. L., Luévano-Martinez, L. A., Chausse, B., and Kowaltowski, A. J. (2017). Caloric restriction increases brain mitochondrial calcium retention capacity and protects against excitotoxicity. Aging Cell 16, 73-81. doi: 10.1111/acel.12527

Andrews, Z. B., Diano, S., and Horvath, T. L. (2005). Mitochondrial uncoupling proteins in the CNS: in support of function and survival. Nat. Rev. Neurosci. 6, 829-840. doi: 10.1038/nrn1767

Ansari, A., Rahman, M. S., Saha, S. K., Saikot, F. K., Deep, A., and Kim, K. H. (2017). Function of the SIRT3 mitochondrial deacetylase in cellular physiology, cancer and neurodegenerative disease. Aging Cell 16, 4-16. doi: 10.1111/acel. 12538

Bao, X., Wang, Y., Li, X., Li, X. M., Liu, Z., Yang, T., et al. (2014). Identification of 'erasers' for lysine crotonylated histone marks using a chemical proteomics approach. Elife 3:e02999. doi: 10.7554/elife.02999

Barreto-Torres, G., Hernandez, J. S., Jang, S. J., Rodríguez-Muñoz, A. R., TorresRamos, C., Basnakian, A. G., et al. (2015). The beneficial effects of AMP kinase activation against oxidative stress are associated with prevention of PPAR $\alpha$ cyclophilin D interaction in cardiomyocytes. Am. J. Physiol. Heart Circ. Physiol. 308, 749-758. doi: 10.1152/ajpheart.00414.2014

Barsoum, M. J., Yuan, H., Gerencser, A. A., Liot, G., Kushnareva, Y., Graber, S., et al. (2006). Nitric oxide-induced mitochondrial fission is regulated by dynamin-related GTPases in neurons. EMBO J. 25, 3900-3911. doi: 10.1038/sj. emboj.7601253

Bauernfeind, A. L., Barks, S. K., Duka, T., Grossman, L. I., Hof, P. R., and Sherwood, C. C. (2014). Aerobic glycolysis in the primate brain: reconsidering the implications for growth and maintenance. Brain Struct. Funct. 219, 1149-1167. doi: 10.1007/s00429-013-0662-z

Bause, A. S., and Haigis, M. C. (2013). SIRT3 regulation of mitochondrial oxidative stress. Exp. Gerontol. 48, 634-639. doi: 10.1016/j.exger.2012.08.007

Bell, E. L., and Guarente, L. (2011). The SirT3 divining rod points to oxidative stress. Mol. Cell 42, 561-568. doi: 10.1016/j.molcel.2011.05.008

Bitterman, K. J., Anderson, R. M., Cohen, H. Y., Latorre-Esteves, M., and Sinclair, D. A. (2002). Inhibition of silencing and accelerated aging by nicotinamide, a putative negative regulator of yeast sir2 and human SIRT1. J. Biol. Chem. 277, 45099-45107. doi: 10.1074/jbc.M205670200

Brachmann, C. B., Sherman, J. M., Devine, S. E., Cameron, E. E., Pillus, L., and Boeke, J. D. (1995). The SIR2 gene family, conserved from bacteria to humans, functions in silencing, cell cycle progression, and chromosome stability. Genes Dev. 9, 2888-2902. doi: 10.1101/gad.9.23.2888

Braidy, N., Poljak, A., Grant, R., Jayasena, T., Mansour, H., Chan-Ling, T., et al. (2015). Differential expression of sirtuins in the aging rat brain. Front. Cell. Neurosci. 9:167. doi: 10.3389/fncel.2015.00167

Brandauer, J., Andersen, M. A., Kellezi, H., Risis, S., Frøsig, C., Vienberg, S. G., et al. (2015). AMP-activated protein kinase controls exercise training- and review, and Mr. Peter Darmos for assistance with manuscript assembly.

\section{SUPPLEMENTARY MATERIAL}

The Supplementary Material for this article can be found online at: https://www.frontiersin.org/articles/10.3389/fncel. 2018.00196/full\#supplementary-material

AICAR-induced increases in SIRT3 and MnSOD. Front. Physiol. 6:85 doi: 10.3389/fphys.2015.00085

Brown, K. D., Maqsood, S., Huang, J. Y., Pan, Y., Harkcom, W., Li, W., et al. (2014). Activation of SIRT3 by the $\mathrm{NAD}^{+}$precursor nicotinamide riboside protects from noise-induced hearing loss. Cell Metab. 20, 1059-1068. doi: 10.1016/j. cmet.2014.11.003

Cantó, C., and Auwerx, J. (2011). Calorie restriction: is AMPK a key sensor and effector? Physiol. 26, 214-224. doi: 10.1152/physiol.00010.2011

Cantó, C., Menzies, K., and Auwerx, J. (2015). NAD ${ }^{+}$metabolism and the control of energy homeostasis - a balancing act between mitochondria and the nucleus. Cell Metab. 22, 31-53. doi: 10.1016/j.cmet.2015.05.023

Chen, C. M. (2011). Mitochondrial dysfunction, metabolic deficits, and increased oxidative stress in Huntington's disease. Chang Gung Med. J. 34, 135-152. doi: 10.1007/978-0-85729-701-3_6

Chen, H., and Chan, D. C. (2009). Mitochondrial dynamics-fusion, fission, movement, and mitophagy-in neurodegenerative diseases. Hum. Mol. Genet. 18, R169-R176. doi: 10.1093/hmg/ddp326

Chen, T., Li, J., Liu, J., Li, N., Wang, S., Liu, H., et al. (2015). Activation of SIRT3 by resveratrol ameliorates cardiac fibrosis and improves cardiac function via the TGF- $\beta /$ Smad3 pathway. Am. J. Physiol. Heart Circ. Physiol. 308, H424-H434. doi: 10.1152/ajpheart.00454.2014

Chen, Y., Zhang, J., Lin, Y., Lei, Q., Guan, K. L., Zhao, S., et al. (2011). Tumour suppressor SIRT3 deacetylates and activates manganese superoxide dismutase to scavenge ROS. EMBO Rep. 12, 534-541. doi: 10.1038/embor.2011.65

Cheng, A., Yang, Y., Zhou, Y., Maharana, C., Lu, D., Peng, W., et al. (2016). Mitochondrial SIRT3 mediates adaptive responses of neurons to exercise and metabolic and excitatory challenges. Cell Metab. 23, 128-142. doi: 10.1016/j. cmet.2015.10.013

Cooper, H. M., and Spelbrink, J. N. (2008). The human SIRT3 protein deacetylase is exclusively mitochondrial. Biochem. J. 411, 279-285. doi: 10.1042/BJ20071624

Cui, X. X., Li, X., Dong, S. Y., Guo, Y. J., Liu, T., and Wu, Y. C. (2017). SIRT3 deacetylated and increased citrate synthase activity in PD model. Biochem. Biophys. Res. Commun. 484, 767-773. doi: 10.1016/j.bbrc.2017. 01.163

Cui, Y., Qin, L., Wu, J., Qu, X., Hou, C., Sun, W., et al. (2015). SIRT3 enhances glycolysis and proliferation in SIRT3-expressing gastric cancer cells. PLoS One 10:e0129834. doi: 10.1371/journal.pone.0129834

Dai, S. H., Chen, T., Li, X., Yue, K. Y., Luo, P., Yang, L. K., et al. (2017). Sirt3 confers protection against neuronal ischemia by inducing autophagy: involvement of the AMPK-mTOR pathway. Free Radic. Biol. Med. 108, 345-353. doi: 10.1016/j. freeradbiomed.2017.04.005

Dai, S. H., Chen, T., Wang, Y. H., Zhu, J., Luo, P., Rao, W., et al. (2014). Sirt3 protects cortical neurons against oxidative stress via regulating mitochondrial $\mathrm{Ca}^{2+}$ and mitochondrial biogenesis. Int. J. Mol. Sci. 15, 14591-14609. doi: 10.3390/ijms150814591

Deslandes, A. (2013). The biological clock keeps ticking, but exercise may turn it back. Arq. Neuropsiquiatr. 71, 113-118. doi: 10.1590/s0004282x2013000200011

Desquiret-Dumas, V., Gueguen, N., Leman, G., Baron, S., Nivet-Antoine, V., Chupin, S., et al. (2013). Resveratrol induces a mitochondrial complex I-dependent increase in NADH oxidation responsible for sirtuin activation in liver cells. J. Biol. Chem. 288, 36662-36675. doi: 10.1074/jbc.M113.466490

Dittenhafer-Reed, K. E., Richards, A. L., Fan, J., Smallegan, M. J., Fotuhi Siahpirani, A., Kemmerer, Z. A., et al. (2015). SIRT3 mediates multi-tissue coupling for metabolic fuel switching. Cell Metab. 21, 637-646. doi: 10.1016/j. cmet.2015.03.007 
Du, J., Zhou, Y., Su, X., Yu, J. J., Khan, S., Jiang, H., et al. (2011). Sirt5 is a NAD-dependent protein lysine demalonylase and desuccinylase. Science 334, 806-809. doi: 10.1126/science.1207861

Edgett, B. A., Hughes, M. C., Matusiak, J. B., Perry, C. G., Simpson, C. A., and Gurd, B. J. (2016). SIRT3 gene expression but not SIRT3 subcellular localization is altered in response to fasting and exercise in human skeletal muscle. Exp. Physiol. 101, 1101-1113. doi: 10.1113/EP085744

Eichner, L. J., and Giguère, V. (2011). Estrogen related receptors (ERRs): a new dawn in transcriptional control of mitochondrial gene networks. Mitochondrion 11, 544-552. doi: 10.1016/j.mito.2011.03.121

Falkowska, A., Gutowska, I., Goschorska, M., Nowacki, P., Chlubek, D., and Baranowska-Bosiacka, I. (2015). Energy metabolism of the brain, including the cooperation between astrocytes and neurons, especially in the context of glycogen metabolism. Int. J. Mol. Sci. 16, 25959-25981. doi: 10.3390/ijms161125939

Federico, A., Cardaioli, E., Da Pozzo, P., Formichi, P., Gallus, G. N., and Radi, E. (2012). Mitochondria, oxidative stress and neurodegeneration. J. Neurol. Sci. 322, 254-262. doi: 10.1016/j.jns.2012.05.030

Fernandez-Marcos, P. J., Jeninga, E. H., Canto, C., Harach, T., de Boer, V. C., Andreux, P., et al. (2012). Muscle or liver-specific Sirt3 deficiency induces hyperacetylation of mitochondrial proteins without affecting global metabolic homeostasis. Sci. Rep. 2:425. doi: 10.1038/srep00425

Ferrer, M. D., Tauler, P., Sureda, A., Tur, J. A., and Pons, A. (2009). Antioxidant regulatory mechanisms in neutrophils and lymphocytes after intense exercise. J. Sports Sci. 27, 49-58. doi: 10.1080/02640410802409683

Finkel, T., Deng, C. X., and Mostoslavsky, R. (2009). Recent progress in the biology and physiology of sirtuins. Nature 460, 587-591. doi: 10.1038/nature08197

Finley, L. W., Haas, W., Desquiret-Dumas, V., Wallace, D. C., Procaccio, V., Gygi, S. P., et al. (2011). Succinate dehydrogenase is a direct target of sirtuin 3 deacetylase activity. PLoS One 6:e23295. doi: 10.1371/journal.pone.0023295

Frahm, T., Mohamed, S. A., Bruse, P., Gemünd, C., Oehmichen, M., and Meissner, C. (2005). Lack of age-related increase of mitochondrial DNA amount in brain, skeletal muscle and human heart. Mech. Ageing Dev. 126, 1192-1200. doi: 10.1016/j.mad.2005.06.008

Frye, R. A. (1999). Characterization of five human cDNAs with homology to the yeast SIR2 gene: Sir2-like proteins (sirtuins) metabolize NAD and may have protein ADP-ribosyltransferase activity. Biochem. Biophys. Res. Commun. 260, 273-279. doi: 10.1006/bbrc.1999.0897

Frye, R. A. (2000). Phylogenetic classification of prokaryotic and eukaryotic Sir2-like proteins. Biochem. Biophys. Res. Commun. 273, 793-798. doi: 10.1006/bbrc. 2000.3000

Fu, J., Jin, J., Cichewicz, R. H., Hageman, S. A., Ellis, T. K., Xiang, L., et al. (2012). trans-(-)-epsilon-Viniferin increases mitochondrial sirtuin 3 (SIRT3), activates AMP-activated protein kinase (AMPK), and protects cells in models of Huntington Disease. J. Biol. Chem. 287, 24460-24472. doi: 10.1074/jbc. M112.382226

Fujino, T., Kondo, J., Ishikawa, M., Morikawa, K., and Yamamoto, T. T. (2001). Acetyl-CoA synthetase 2, a mitochondrial matrix enzyme involved in the oxidation of acetate. J. Biol. Chem. 276, 11420-11426. doi: 10.1074/jbc. M008782200

Fuke, S., Kubota-Sakashita, M., Kasahara, T., Shigeyoshi, Y., and Kato, T. (2011). Regional variation in mitochondrial DNA copy number in mouse brain. Biochim. Biophys. Acta 1807, 270-274. doi: 10.1016/j.bbabio.2010.11.016

Gertz, M., Nguyen, G. T., Fischer, F., Suenkel, B., Schlicker, C., Fränzel, B., et al. (2012). A molecular mechanism for direct sirtuin activation by resveratrol. PLoS One 7:e49761. doi: 10.1371/journal.pone.0049761

Giralt, A., Hondares, E., Villena, J. A., Ribas, F., Díaz-Delfín, J., Giralt, M., et al. (2011). Peroxisome proliferator-activated receptor- $\gamma$ coactivator- $1 \alpha$ controls transcription of the Sirt3 gene, an essential component of the thermogenic brown adipocyte phenotype. J. Biol. Chem. 286, 16958-16966. doi: 10.1074/jbc. M110.202390

Gleave, J. A., Arathoon, L. R., Trinh, D., Lizal, K. E., Giguere, N., Barber, J. H. M., et al. (2017). Sirtuin 3 rescues neurons through the stabilisation of mitochondrial biogenetics in the virally-expressing mutant $\alpha$-synuclein rat model of parkinsonism. Neurobiol. Dis. 106, 133-146. doi: 10.1016/j.nbd.2017. 06.009

Glenn, T. C., Martin, N. A., Horning, M. A., McArthur, D. L., Hovda, D. A., Vespa, P., et al. (2015). Lactate: brain fuel in human traumatic brain injury: a comparison with normal healthy control subjects. J. Neurotrauma 32, 820-832. doi: 10.1089/neu.2014.3483

Grimm, A., Friedland, K., and Eckert, A. (2016). Mitochondrial dysfunction: the missing link between aging and sporadic Alzheimer's disease. Biogerontology 17, 281-296. doi: 10.1007/s10522-015-9618-4

Guan, X., Lin, P., Knoll, E., and Chakrabarti, R. (2014). Mechanism of inhibition of the human sirtuin enzyme SIRT3 by nicotinamide: computational and experimental studies. PLoS One 9:e107729. doi: 10.1371/journal.pone. 0107729

Guo, C., Sun, L., Chen, X., and Zhang, D. (2013). Oxidative stress, mitochondrial damage and neurodegenerative diseases. Neural Regen. Res. 8, 2003-2014. doi: 10.3969/j.issn.1673-5374.2013.21.009

Gurd, B. J., Holloway, G. P., Yoshida, Y., and Bonen, A. (2012). In mammalian muscle, SIRT3 is present in mitochondria and not in the nucleus; and SIRT3 is upregulated by chronic muscle contraction in an adenosine monophosphateactivated protein kinase-independent manner. Metabolism 61, 733-741. doi: 10.1016/j.metabol.2011.09.016

Hafner, A. V., Dai, J., Gomes, A. P., Xiao, C. Y., Palmeira, C. M., Rosenzweig, A., et al. (2010). Regulation of the mPTP by SIRT3-mediated deacetylation of CypD at lysine 166 suppresses age-related cardiac hypertrophy. Aging 2, 914-923. doi: 10.18632/aging.100252

Halestrap, A. P., and Pasdois, P. (2009). The role of the mitochondrial permeability transition pore in heart disease. Biochim. Biophys. Acta 1787, 1402-1415. doi: 10.1016/j.bbabio.2008.12.017

Hallows, W. C., Lee, S., and Denu, J. M. (2006). Sirtuins deacetylate and activate mammalian acetyl-CoA synthetases. Proc. Natl. Acad. Sci. U S A 103, 10230-10235. doi: 10.1073/pnas.0604392103

Hallows, W. C., Yu, W., Smith, B. C., Devries, M. K., Ellinger, J. J., Someya, S., et al. (2011). Sirt3 promotes the urea cycle and fatty acid oxidation during dietary restriction. Mol. Cell 41, 139-149. doi: 10.1016/j.molcel.2011. 01.002

Han, P., Tang, Z., Yin, J., Maalouf, M., Beach, T. G., Reiman, E. M., et al. (2014). Pituitary adenylate cyclase-activating polypeptide protects against $\beta$-amyloid toxicity. Neurobiol. Aging 35, 2064-2071. doi: 10.1016/j.neurobiolaging.2014. 03.022

Harlan, B. A., Pehar, M., Sharma, D. R., Beeson, G., Beeson, C. C., and Vargas, M. R. (2016). Enhancing $\mathrm{NAD}^{+}$salvage pathway reverts the toxicity of primary astrocytes expressing amyotrophic lateral sclerosis-linked mutant superoxide dismutase 1 (SOD1). J. Biol. Chem. 291, 10836-10846. doi: 10.1074/jbc.M115.698779

Hebert, A. S., Dittenhafer-Reed, K. E., Yu, W., Bailey, D. J., Selen, E. S., Boersma, M. D., et al. (2013). Calorie restriction and SIRT3 trigger global reprogramming of the mitochondrial protein acetylome. Mol. Cell 49, 186-199. doi: 10.1016/j.molcel.2012.10.024

Hipkiss, A. R. (2008). Energy metabolism, altered proteins, sirtuins and ageing: converging mechanisms? Biogerontology 9, 49-55. doi: 10.1007/s10522-0079110-x

Hirschey, M. D., Shimazu, T., Goetzman, E., Jing, E., Schwer, B., Lombard, D. B., et al. (2010). SIRT3 regulates mitochondrial fatty-acid oxidation by reversible enzyme deacetylation. Nature 464, 121-125. doi: 10.1038/nature08778

Hirschey, M. D., Shimazu, T., Huang, J. Y., Schwer, B., and Verdin, E. (2011). SIRT3 regulates mitochondrial protein acetylation and intermediary metabolism. Cold Spring Harb. Symp. Quant. Biol. 76, 267-277. doi: 10.1101/sqb.2011.76.010850

Hokari, F., Kawasaki, E., Sakai, A., Koshinaka, K., Sakuma, K., and Kawanaka, K. (2010). Muscle contractile activity regulates Sirt3 protein expression in rat skeletal muscles. J. Appl. Physiol. 109, 332-340. doi: 10.1152/japplphysiol. 00335.2009

Howitz, K. T., Bitterman, K. J., Cohen, H. Y., Lamming, D. W., Lavu, S., Wood, J. G., et al. (2003). Small molecule activators of sirtuins extend Saccharomyces cerevisiae lifespan. Nature 425, 191-196. doi: 10.1038/ nature 01960

Ivy, J. M., Klar, A. J., and Hicks, J. B. (1986). Cloning and characterization of four SIR genes of Saccharomyces cerevisiae. Mol. Cell. Biol. 6, 688-702. doi: $10.1128 / \mathrm{mcb} \cdot 6.2 .688$

Iwahara, T., Bonasio, R., Narendra, V., and Reinberg, D. (2012). SIRT3 functions in the nucleus in the control of stress-related gene expression. Mol. Cell. Biol. 32, 5022-5034. doi: 10.1128/MCB.00822-12 
Jacobs, K. M., Pennington, J. D., Bisht, K. S., Aykin-Burns, N., Kim, H. S., Mishra, M., et al. (2008). SIRT3 interacts with the daf-16 homolog FOXO3a in the mitochondria, as well as increases FOXO3a dependent gene expression. Int. J. Biol. Sci. 4, 291-299. doi: 10.7150/ijbs.4.291

Jiang, H., Khan, S., Wang, Y., Charron, G., He, B., Sebastian, C., et al. (2013). SIRT6 regulates TNF- $\alpha$ secretion through hydrolysis of long-chain fatty acyl lysine. Nature 496, 110-113. doi: 10.1038/nature12038

Jiang, Z., Wang, W., Perry, G., Zhu, X., and Wang, X. (2015). Mitochondrial dynamic abnormalities in amyotrophic lateral sclerosis. Transl. Neurodegener. 4:14. doi: 10.1186/s40035-015-0037-x

Jin, L., Galonek, H., Israelian, K., Choy, W., Morrison, M., Xia, Y., et al. (2009). Biochemical characterization, localization, and tissue distribution of the longer form of mouse SIRT3. Protein Sci. 18, 514-525. doi: 10.1002/pro.50

Jing, E., O’Neill, B. T., Rardin, M. J., Kleinridders, A., Ilkeyeva, O. R., Ussar, S., et al. (2013). Sirt3 regulates metabolic flexibility of skeletal muscle through reversible enzymatic deacetylation. Diabetes 62, 3404-3417. doi: 10.2337/ db12-1650

Kaddour-Djebbar, I., Choudhary, V., Brooks, C., Ghazaly, T., Lakshmikanthan, V., Dong, Z., et al. (2010). Specific mitochondrial calcium overload induces mitochondrial fission in prostate cancer cells. Int. J. Oncol. 36, 1437-1444. doi: 10.3892/ijo_00000629

Kaeberlein, M., McVey, M., and Guarente, L. (1999). The SIR2/3/4 complex and SIR2 alone promote longevity in Saccharomyces cerevisiae by two different mechanisms. Genes Dev. 13, 2570-2580. doi: 10.1101/gad.13.19.2570

Kendrick, A. A., Choudhury, M., Rahman, S. M., McCurdy, C. E., Friederich, M., Van Hove, J. L., et al. (2011). Fatty liver is associated with reduced SIRT3 activity and mitochondrial protein hyperacetylation. Biochem. J. 433, 505-514. doi: 10.1042/BJ20100791

Kim, S., Benguria, A., Lai, C. Y., and Jazwinski, S. M. (1999). Modulation of life-span by histone deacetylase genes in Saccharomyces cerevisiae. Mol. Biol. Cell 10, 3125-3136. doi: 10.1091/mbc.10.10.3125

Kim, G. H., Kim, J. E., Rhie, S. J., and Yoon, S. (2015). The role of oxidative stress in neurodegenerative diseases. Exp. Neurobiol. 24, 325-340. doi: 10.5607/en.2015. 24.4.325

Kim, S. H., Lu, H. F., and Alano, C. C. (2011). Neuronal Sirt3 protects against excitotoxic injury in mouse cortical neuron culture. PLoS One 6:e14731. doi: 10.1371/journal.pone.0014731

Kim, H. S., Patel, K., Muldoon-Jacobs, K., Bisht, K. S., Aykin-Burns, N., Pennington, J. D., et al. (2010). SIRT3 is a mitochondria-localized tumor suppressor required for maintenance of mitochondrial integrity and metabolism during stress. Cancer Cell 17, 41-52. doi: 10.1016/j.ccr.2009.11.023

Klingenspor, M. (2003). Cold-induced recruitment of brown adipose tissue thermogenesis. Exp. Physiol. 88, 141-148. doi: 10.1113/eph8802508

Kobayashi, M., Takeda, K., Narita, T., Nagai, K., Okita, N., Sudo, Y., et al. (2017). Mitochondrial intermediate peptidase is a novel regulator of sirtuin-3 activation by caloric restriction. FEBS Lett. 591, 4067-4073. doi: 10.1002/18733468.12914

Kong, X., Wang, R., Xue, Y., Liu, X., Zhang, H., Chen, Y., et al. (2010). Sirtuin 3 , a new target of PGC- $1 \alpha$, plays an important role in the suppression of ROS and mitochondrial biogenesis. PLoS One 5:e11707. doi: 10.1371/journal.pone. 0011707

Kupis, W., Palyga, J., Tomal, E., and Niewiadomska, E. (2016). The role of sirtuins in cellular homeostasis. J. Physiol. Biochem. 72, 371-380. doi: 10.1007/s13105016-0492-6

Lai, Y. C., Tabima, D. M., Dube, J. J., Hughan, K. S., Vanderpool, R. R., Goncharov, D. A., et al. (2016). SIRT3-AMP-activated protein kinase activation by nitrite and metformin improves hyperglycemia and normalizes pulmonary hypertension associated with heart failure with preserved ejection fraction. Circulation 133, 717-731. doi: 10.1161/CIRCULATIONAHA.115. 018935

Landry, J., Sutton, A., Tafrov, S. T., Heller, R. C., Stebbins, J., Pillus, L., et al. (2000). The silencing protein SIR2 and its homologs are NAD-dependent protein deacetylases. Proc. Natl. Acad. Sci. U S A 97, 5807-5811. doi: 10.1073/pnas. 110148297

Law, I. K., Liu, L., Xu, A., Lam, K. S., Vanhoutte, P. M., Che, C. M., et al. (2009). Identification and characterization of proteins interacting with SIRT1 and SIRT3: implications in the anti-aging and metabolic effects of sirtuins. Proteomics 9, 2444-2456. doi: 10.1002/pmic.200800738
Lenard, N. R., and Berthoud, H. R. (2008). Central and peripheral regulation of food intake and physical activity: pathways and genes. Obesity 16, S11-S22. doi: 10.1038/oby.2008.511

Li, P. A., Hou, X., and Hao, S. (2017). Mitochondrial biogenesis in neurodegeneration. J. Neurosci. Res. 95, 2025-2029. doi: 10.1002/jnr. 24042

Li, T. T., Li, Z. H., Zhuang, X. Y., and Fu, Y. C. (2011). Effects of caloric restriction on the oxidative stress injury and the expression of SIRT3 in PC12 cell. Zhonghua Yi Xue Za Zhi 91, 350-358. doi: 10.3760/cma.j.issn.03762491.2011.05.016

Liang, F., Wang, X., Ow, S. H., Chen, W., and Ong, W. C. (2017). Sirtuin 5 is anti-apoptotic and anti-oxidative in cultured SH-EP neuroblastoma cells. Neurotox. Res. 31, 63-76. doi: 10.1007/s12640-016-9664-y

Liu, B., Che, W., Xue, J., Zheng, C., Tang, K., Zhang, J., et al. (2013). Sirt4 prevents hypoxia-induced apoptosis in H9c2 cardiomyoblast cells. Cell. Physiol. Biochem. 32, 655-662. doi: 10.1159/000354469

Liu, L., Peritore, C., Ginsberg, J., Kayhan, M., and Donmez, G. (2015). SIRT3 attenuates MPTP-induced nigrostriatal degeneration via enhancing mitochondrial antioxidant capacity. Neurochem. Res. 40, 600-608. doi: 10.1007/s11064-014-1507-8

Liu, L., Sanosaka, M., Lei, S., Bestwick, M. L., Frey, J. H. Jr., Surovtseva, Y. V., et al. (2011). LRP130 protein remodels mitochondria and stimulates fatty acid oxidation. J. Biol. Chem. 286, 41253-41264. doi: 10.1074/jbc.M111.276121

Liszt, G., Ford, E., Kurtev, M., and Guarente, L. (2005). Mouse Sir2 homolog SIRT6 is a nuclear ADP-ribosyltransferase. J. Biol. Chem. 280, 21313-21320. doi: 10.1074/jbc.M413296200

Lombard, D. B., Alt, F. W., Cheng, H. L., Bunkenborg, J., Streeper, R. S., Mostoslavsky, R., et al. (2007). Mammalian Sir2 homolog SIRT3 regulates global mitochondrial lysine acetylation. Mol. Cell. Biol. 27, 8807-8814. doi: $10.1128 / \mathrm{mcb} .01636-07$

Lonze, B. E., and Ginty, D. D. (2002). Function and regulation of CREB family transcription factors in the nervous system. Neuron 35, 605-623. doi: 10.1016/s0896-6273(02)00828-0

Lu, J., Zhang, H., Chen, X., Zou, Y., Li, J., Wang, L., et al. (2017). A small molecule activator of SIRT3 promotes deacetylation and activation of manganese superoxide dismutase. Free Radic. Biol. Med. 112, 287-297. doi: 10.1016/j. freeradbiomed.2017.07.012

Magnifico, S., Saias, L., Deleglise, B., Duplus, E., Kilinc, D., Miquel, M. C., et al. (2013). NAD + acts on mitochondrial SirT3 to prevent axonal caspase activation and axonal degeneration. FASEB J. 27, 4712-4722. doi: 10.1096/fj.13-229781

Marmorstein, R. (2004). Structure and chemistry of the Sir2 family of NAD+dependent histone/protein deactylases. Biochem. Soc. Trans. 32, 904-909. doi: 10.1042/bst0320904

Martin, L. J. (2012). Biology of mitochondria in neurodegenerative diseases. Prog. Mol. Biol. Transl. Sci. 107, 355-415. doi: 10.1016/b978-0-12-385883-2. 00005-9

Mathias, R. A., Greco, T. M., Oberstein, A., Budayeva, H. G., Chakrabarti, R., Rowland, E. A., et al. (2014). Sirtuin 4 is a lipoamidase regulating pyruvate dehydrogenase complex activity. Cell 159, 1615-1625. doi: 10.1016/j.cell.2014. 11.046

McInerny, S. C., Brown, A. L., and Smith, D. W. (2009). Region-specific changes in mitochondrial D-loop in aged rat CNS. Mech. Ageing Dev. 130, 343-349. doi: 10.1016/j.mad.2009.01.008

Mencarelli, C., and Martinez-Martinez, P. (2013). Ceramide function in the brain: when a slight tilt is enough. Cell. Mol. Life Sci. 70, 181-203. doi: 10.1007/s00018012-1038-x

Mergenthaler, P., Lindauer, U., Dienel, G. A., and Meisel, A. (2013). Sugar for the brain: the role of glucose in physiological and pathological brain function. Trends Neurosci. 36, 587-597. doi: 10.1016/j.tins.2013.07.001

Michan, S. (2014). Calorie restriction and $\mathrm{NAD}^{+} /$sirtuin counteract the hallmarks of aging. Front. Biosci. 19, 1300-1319. doi: 10.2741/4283

Michishita, E., McCord, R. A., Berber, E., Kioi, M., Padilla-Nash, H., Damian, M., et al. (2008). SIRT6 is a histone H3 lysine 9 deacetylase that modulates telomeric chromatin. Nature 452, 492-496. doi: 10.1038/nature06736

Michishita, E., Park, J. Y., Burneskis, J. M., Barrett, J. C., and Horikawa, I. (2005). Evolutionarily conserved and nonconserved cellular localizations and functions of human SIRT proteins. Mol. Biol. Cell. 16, 4623-4635. doi: 10.1091/mbc.e0501-0033 
Milone, M., and Benarroch, E. E. (2012). Mitochondrial dynamics: general concepts and clinical implications. Neurology 78, 1612-1619. doi: 10.1212/WNL.0b013e3182563c46

Min, J., Landry, J., Sternglanz, R., and Xu, R. M. (2001). Crystal structure of a SIR2 homolog-NAD complex. Cell 105, 269-279. doi: 10.1016/S00928674(01)00317-8

Mishra, P., and Chan, D. C., (2016). Metabolic regulation of mitochondrial dynamics. J. Cell. Biol. 212, 379-387. doi: 10.1083/jcb.201511036

Mootha, V. K., Handschin, C., Arlow, D., Xie, X., St. Pierre, J., Sihag, S., et al. (2004). Err $\alpha$ and Gabpa/b specify PGC-1 $\alpha$-dependent oxidative phosphorylation gene expression that is altered in diabetic muscle. Proc. Natl. Acad. Sci. U S A 101, 6570-6575. doi: 10.1073/pnas.0401401101

Morigi, M., Perico, L., Rota, C., Longaretti, L., Conti, S., Rottoli, D., et al. (2015). Sirtuin 3-dependent mitochondrial dynamic improvements protect against acute kidney injury. J. Clin. Invest. 125, 715-726. doi: 10.1172/jci77632

Morris, G., and Berk, M. (2015). The many roads to mitochondrial dysfunction in neuroimmune and neuropsychiatric disorders. BMC Med. 13:68. doi: 10.1186/s12916-015-0310-y

Mostoslavsky, R., Chua, K. F., Lombard, D. B., Pang, W. W., Fischer, M. R., Gellon, L., et al. (2006). Genomic instability and aging-like phenotype in the absence of mammalian SIRT6. Cell 124, 315-329. doi: 10.1016/j.cell.2005.11.044

Nakamura, Y., Ogura, M., Tanaka, D., and Inagaki, N. (2008). Localization of mouse mitochondrial SIRT proteins: shift of SIRT3 to nucleus by co-expression with SIRT5. Biochem. Biophys. Res. Commun. 366, 174-179. doi: 10.1016/j.bbrc. 2007.11.122

Nguyen, G. T., Gertz, M., and Steegborn, C. (2013). Crystal structures of Sirt3 complexes with $4^{\prime}$-bromo-resveratrol reveal binding sites and inhibition mechanism. Chem. Biol. 20, 1375-1385. doi: 10.1016/j.chembiol.2013. 09.019

Nguyen, T. T. M., Wong, R., Menazza, S., Sun, J., Chen, Y., Wang, G., et al. (2013). Cyclophilin D modulates the mitochondrial acetylome. Circ. Res. 113, 1308-1319. doi: 10.1161/CIRCRESAHA.113.301867

North, B. J., Marshall, B. L., Borra, M. T., Denu, J. M., and Verdin, E. (2003). The human Sir2 ortholog, SIRT2, is an $\mathrm{NAD}^{+}$-dependent tubulin deacetylase. Mol. Cell 11, 437-444. doi: 10.1016/s1097-2765(03)00038-8

Novgorodov, S. A., and Gudz, T. I. (2011). Ceramide and mitochondria in ischemic brain injury. Int. J. Biochem. Mol. Biol. 2, 347-361.

Novgorodov, S. A., Riley, C. L., Keffler, J. A., Yu, J., Kindy, M. S., Macklin, D. B., et al. (2016). SIRT3 Deacetylates Ceramide Synthases. Implications for mitochondrial dysfunction and brain injury. J. Biol. Chem. 291, 1957-1973. doi: 10.1074/jbc.M115.668228

Onyango, P., Celic, I., McCaffery, J. M., Boeke, J. D., and Feinberg, A. P. (2002). SIRT3, a human SIR2 homologue, is an NAD-dependent deacetylase localized to mitochondria. Proc. Natl. Acad. Sci. U S A 99, 13653-13658. doi: 10.1073/pnas.222538099

Onyango, I. G., Lu, J., Rodova, M., Lezi, E., Crafter, A. B., and Swerdlow, R. H. (2010). Regulation of neuron mitochondrial biogenesis and relevance to brain health. Biochim. Biophys. Acta 1802, 228-234. doi: 10.1016/j.bbadis.2009. 07.014

Ozden, O., Park, S. H., Wagner, B. A., Song, H. Y., Zhu, Y., Vassilopoulos, A., et al. (2014). SIRT3 deacetylates and increases pyruvate dehydrogenase activity in cancer cells. Free Radic. Biol. Med. 76, 163-172. doi: 10.1016/j.freeradbiomed. 2014.08.001

Palacios, O. M., Carmona, J. J., Michan, S., Chen, K. Y., Manabe, Y., Ward, J. L., et al. (2009). Diet and exercise signals regulate SIRT3 and activate AMPK and PGC-1 $\alpha$ in skeletal muscle. Aging 1, 771-783. doi: 10.18632/aging. 100075

Parihar, P., Solanki, I., Mansuri, M. L., and Parihar, M. S. (2015). Mitochondrial sirtuins: emerging roles in metabolic regulations, energy homeostasis and diseases. Exp. Gerontol. 61, 130-141. doi: 10.1016/j.exger.2014.12.004

Parodi-Rullan, R. M., Barreto-Torres, G., Ruiz, L., Casasnovas, J., and Javadov, S. (2012). Direct renin inhibition exerts an anti-hypertrophic effect associated with improved mitochondrial function in post-infarction heart failure in diabetic rats. Cell. Physiol. Biochem. 29, 841-850. doi: 10.1159/000 178526

Parodi-Rullam, R. M., Chapa-Dubocq, X., Rullan, P. J., Jang, S., and Javadov, S. (2017). High sensitivity of SIRT3 deficient hearts to ischemia-reperfusion is associated with mitochondrial abnormalities. Front. Pharmacol. 8:275. doi: 10.3389/fphar.2017.00275

Patel, K., Sherrill, J., Mrksich, M., and Scholle, M. D. (2015). Discovery of SIRT3 inhibitors using SAMDI mass spectrometry. J. Biomol. Screen. 20, 842-848. doi: 10.1177/1087057115588512

Pellerin, L., Bergersen, L. H., Halestrap, A. P., and Pierre, K. (2005). Cellular and subcellular distribution of monocarboxylate transporters in cultured brain cells and in the adult brain. J. Neurosci. Res. 79, 55-64. doi: 10.1002/jnr.20307

Peng, C., Lu, Z., Xie, Z., Cheng, Z., Chen, Y., Tan, M., et al. (2011). The first identification of lysine malonylation substrates and its regulatory enzyme. Mol. Cell. Proteomics 10:M111.012658. doi: 10.1074/mcp.M111. 012658

Perrod, S., Cockell, M. M., Laroche, T., and Renauld, H. (2001). A cytosolic NAD-dependent deacetylase, Hst2p, can modulate nucleolar and telomeric silencing in yeast. EMBO J. 20, 197-209. doi: 10.1093/emboj/20. 1.197

Pfister, J. A., Ma, C., Morrison, B. E., and D’Mello, S. R. (2008). Opposing effects of sirtuins on neuronal survival: SIRT1-mediated neuroprotection is independent of its deacetylase activity. PLoS One 3:e4090. doi: 10.1371/journal.pone. 0004090

Pillai, V. B., Bindu, S., Sharp, W., Fang, Y. H., Kim, G., Gupta, M., et al. (2016). Sirt3 protects mitochondrial DNA damage and blocks the development of doxorubicin-induced cardiomyopathy in mice. Am. J. Physiol. Heart Circ. Physiol. 310, H962-H972. doi: 10.1152/ajpheart. 00832.2015

Pillai, V. B., Samant, S., Sundaresan, N. R., Raghuraman, H., Kim, G., Bonner, M. Y., et al. (2015). Honokiol blocks and reverses cardiac hypertrophy in mice by activating mitochondrial Sirt3. Nat. Commun. 6:6656. doi: $10.1038 /$ ncomms7656

Prins, M. L. (2012). Cerebral ketone metabolism during development and injury. Epilepsy Res. 100, 218-223. doi: 10.1016/j.eplepsyres.2011.09.027

Proia, P., Di Liegro, C. M., Schiera, G., Fricano, A., and Di Liegro, I. (2016). Lactate as a metabolite and a regulator in the central nervous system. Int. J. Mol. Sci. 17:E1450. doi: 10.3390/ijms17091450

Qiu, X., Brown, K., Hirschey, M. D., Verdin, E., and Chen, D. (2010). Calorie restriction reduces oxidative stress by SIRT3-mediated SOD2 activation. Cell Metab. 12, 662-667. doi: 10.1016/j.cmet.2010.11.015

Radak, Z., Suzuki, K., Higuchi, M., Balogh, L., Boldogh, I., and Koltai, E. (2016). Physical exercise, reactive oxygen species and neuroprotection. Free Radic. Biol. Med. 98, 187-196. doi: 10.1016/j.freeradbiomed.2016.01.024

Rahman, M., Nirala, N. K., Singh, A., Zhu, L. J., Taguchi, K., Bamba, T., et al. (2014). Drosophila Sirt2/mammalian SIRT3 deacetylates ATP synthase $\beta$ and regulates complex V activity. J. Cell Biol. 206, 289-305. doi: 10.1083/jcb. 201404118

Ramesh, S., Govindarajulu, M., Lynd, T., Briggs, G., Adamek, D., Jones, E., et al. (2018). SIRT3 activator Honokiol attenuates $\beta$-Amyloid by modulating amyloidogenic pathway. PLoS One 13:e0190350. doi: 10.1371/journal.pone. 0190350

Rao, V. K., Carlson, E. A., and Yan, S. S. (2014). Mitochondrial permeability transition pore is a potential drug target for neurodegeneration. Biochim. Biophys. Acta 1842, 1267-1272. doi: 10.1016/j.bbadis.2013.09.003

Ren, J. H., Chen, X., Zhou, L., Tao, N. N., Zhou, H. Z., Liu, B., et al. (2016). Protective role of sirtuin3 (SIRT3) in oxidative stress mediated by hepatitis B virus X protein expression. PLoS One 11:e0150961. doi: 10.1371/journal.pone. 0150961

Richter, E. A., and Ruderman, N. B. (2009). AMPK and the biochemistry of exercise: implications for human health and disease. Biochem. J. 418, 261-275. doi: 10.1042/BJ20082055

Rine, J., and Herskowitz, I. (1987). Four genes responsible for a position effect on expression from HML and HMR in Saccharomyces cerevisiae. Genetics 116, 9-22.

Salo, H. S., Laitinen, T., Poso, A., Jarho, E., and Lahtela-Kakkonen, M. (2013). Identification of novel SIRT3 inhibitor scaffolds by virtual screening. Bioorg. Med. Chem. Lett. 23, 2990-2995. doi: 10.1016/j.bmcl.2013. 03.033

Salvatori, I., Valle, C., Ferri, A., and Carri, M. T. (2017). SIRT3 and mitochondrial metabolism in neurodegenerative diseases. Neurochem. Int. 109, 184-192. doi: 10.1016/j.neuint.2017.04.012 
Samant, S. A., Zhang, H. J., Hong, Z., Pillai, V. B., Sundaresan, N. R., Wolfgeher, D., et al. (2014). SIRT3 deacetylates and activates OPA1 to regulate mitochondrial dynamics during stress. Mol. Cell. Biol. 34, 807-819. doi: $10.1128 / \mathrm{mcb} .01483-13$

Satterstrom, F. K., Swindell, W. R., Laurent, G., Vyas, S., Bulyk, M. L., Haigis, M. C., et al. (2016). Metabolic regulation of mitochondrial dynamics. Aging Cell. 14, 818-825. doi: 10.1111/acel.12360

Scher, M. B., Vaquero, A., and Reinberg, D. (2007). SirT3 is a nuclear $\mathrm{NAD}^{+}$. dependent histone deacetylase that translocates to the mitochondria upon cellular stress. Genes Dev. 21, 920-928. doi: 10.1101/gad.1527307

Schirmer, H., Pereira, T. C., Rico, E. P., Rosemberg, D. B., Bonan, C. D., Bogo, M. R., et al. (2012). Modulatory effect of resveratrol on SIRT1, SIRT3, SIRT4, PGC1 $\alpha$ and NAMPT gene expression profiles in wild-type adult zebrafish liver. Mol. Biol. Rep. 39, 3281-3289. doi: 10.1007/s11033-0111096-4

Schlicker, C., Gertz, M., Papatheodorou, P., Kachholz, B., Becker, C. F., and Steegborn, C. (2008). Substrates and regulation mechanisms for the human mitochondrial sirtuins Sirt3 and Sirt5. J. Mol. Biol. 382, 790-801. doi: 10.1016/j. jmb.2008.07.048

Schurov, I. L., McNulty, S., Best, J. D., Sloper, P. J., and Hastings, M. H. (1999). Glutamatergic induction of CREB phosphorylation and Fos expression in primary cultures of the suprachiasmatic hypothalamus in vitro is mediated by co-ordinate activity of NMDA and non-NMDA receptors. J. Neuroendocrinol. 11, 43-51. doi: 10.1046/j.1365-2826.1999.00289.x

Schwer, B., North, B. J., Frye, R. A., Ott, M., and Verdin, E. (2002). The human silent information regulator (Sir)2 homologue hSIRT3 is a mitochondrial nicotinamide adenine dinucleotide-dependent deacetylase. J. Cell Biol. 158, 647-657. doi: 10.1083/jcb.200205057

Shi, H., Deng, H. X., Gius, D., Schumacker, P. T., Surmeier, D. J., and Ma, Y. C. (2017). Sirt3 protects dopaminergic neurons from mitochondrial oxidative stress. Hum. Mol. Genet. 26, 1915-1926. doi: 10.1093/hmg/ ddx 100

Shi, T., Wang, F., Stieren, E., and Tong, Q. (2005). SIRT3, a mitochondrial sirtuin deacetylase, regulates mitochondrial function and thermogenesis in brown adipocytes. J. Biol. Chem. 280, 13560-13567. doi: 10.1074/jbc.M414 670200

Shimazu, T., Hirschey, M. D., Hua, L., Dittenhafer-Reed, K. E., Schwer, B., Lombard, D. B., et al. (2010). SIRT3 deacetylates mitochondrial 3-hydroxy3-methylglutaryl CoA synthase 2 and regulates ketone body production. Cell Metab. 12, 654-661. doi: 10.1016/j.cmet.2010.11.003

Shore, D., Squire, M., and Nasmyth, K. A. (1984). Characterization of two genes required for the position-effect control of yeast mating-type genes. EMBO J. 3, 2817-2823.

Shulyakova, N., Sidorova-Darmos, E., Fong, J., Zhang, G., Mills, L. R., and Eubanks, J. H. (2014). Over-expression of the Sirt3 sirtuin Protects neuronally differentiated PC12 Cells from degeneration induced by oxidative stress and trophic withdrawal. Brain Res. 1587, 40-53. doi: 10.1016/j.brainres.2014.08.066

Sidorova-Darmos, E., Wither, R. G., Shulyakova, N., Fisher, C., Ratnam, M., Aarts, M., et al. (2014). Differential expression of sirtuin family members in the developing, adult and aged rat brain. Front. Aging Neurosci. 6:333. doi: $10.3389 /$ fnagi.2014.00333

Sims, N. R., and Muyderman, H. (2010). Mitochondria, oxidative metabolism and cell death in stroke. Biochim. Biophys. Acta 1802, 80-91. doi: 10.1016/j.bbadis. 2009.09.003

Singh, N., Agrawal, M., and Doré, S. (2013). Neuroprotective properties and mechanisms of resveratrol in in vitro and in vivo experimental cerebral stroke models. ACS Chem. Neurosci. 4, 1151-1162. doi: 10.1021/cn400094w

Someya, S., Yu, W., Hallows, W. C., Xu, J., Vann, J. M., Leeuwenburgh, C., et al. (2010). Sirt3 mediates reduction of oxidative damage and prevention of age-related hearing loss under caloric restriction. Cell 143, 802-812. doi: 10.1016/j.cell.2010.10.002

Song, W., Song, Y., Kincaid, B., Bossy, B., and Bossy-Wetzel, E. (2013). Mutant SOD1G93A triggers mitochondrial fragmentation in spinal cord motor neurons: neuroprotection by SIRT3 and PGC-1 $\alpha$. Neurobiol. Dis. $51,72-81$. doi: 10.1016/j.nbd.2012.07.004

Steinman, Q. M., Gao, V., and Alberini, C. M. (2016). The role of lactate-mediated metabolic coupling between astrocytes and neurons in long-term memory formation. Front. Integr. Neurosci. 10:10. doi: 10.3389/fnint.2016.00010
Stram, A. R., and Payne, R. M. (2016). Post-translational modifications in mitochondria: protein signaling in the powerhouse. Cell. Mol. Life Sci. 73, 4063-4073. doi: 10.1007/s00018-016-2280-4

Sullivan, P. G., Springer, J. E., Hall, E. D., and Scheff, S. W. (2004). Mitochondrial uncoupling as a therapeutic target following neuronal injury. J. Bioenerg. Biomembr. 36, 353-356. doi: 10.1023/b:jobb.0000041767. 30992.19

Sun, A. Y., Wang, Q., Simonyi, A., and Sun, G. Y. (2010). Resveratrol as a therapeutic agent for neurodegenerative diseases. Mol. Neurobiol. 41, 375-383. doi: 10.1007/s12035-010-8111-y

Sundaresan, N. R., Samant, S. A., Pillai, V. B., Rajamohan, S. B., and Gupta, M. P. (2008). SIRT3 is a stress-responsive deacetylase in cardiomyocytes that protects cells from stress-mediated cell death by deacetylation of Ku70. Mol. Cell. Biol. 28, 6384-6401. doi: 10.1128/mcb.00426-08

Tan, M., Peng, C., Anderson, K. A., Chhoy, P., Xie, Z., Dai, L., et al. (2014). Lysine glutarylation is a protein posttranslational modification regulated by SIRT5. Cell Metab. 19, 605-617. doi: 10.1016/j.cmet.2014.03.014

Tanner, K. G., Landry, J., Sternglanz, R., and Denu, J. M. (2000). Silent information regulator 2 family of NAD- dependent histone/protein deacetylases generates a unique product, 1-O-acetyl-ADP-ribose. Proc. Natl. Acad. Sci. U S A 97, 14178-14182. doi: 10.1073/pnas.250422697

Tao, R., Coleman, M. C., Pennington, J. D., Ozden, O., Park, S. H., Jiang, H., et al. (2010). Sirt3-mediated deacetylation of evolutionarily conserved lysine 122 regulates MnSOD activity in response to stress. Mol. Cell 40, 893-904. doi: 10.1016/j.molcel.2010.12.013

Tauriainen, E., Luostarinen, M., Martonen, E., Finckenberg, P., Kovalainen, M., Huotari, A., et al. (2011). Distinct effects of calorie restriction and resveratrol on diet-induced obesity and Fatty liver formation. J. Nutr. Metab. 2011:525094. doi: 10.1155/2011/525094

Thomson, D. M., Herway, S. T., Fillmore, N., Kim, H., Brown, J. D., Barrow, J. R., et al. (2008). AMP-activated protein kinase phosphorylates transcription factors of the CREB family. J. Appl. Physiol. 104, 429-438. doi: 10.1152/japplphysiol.00900.2007

Tissenbaum, H. A., and Guarente, L. (2001). Increased dosage of a sir-2 gene extends lifespan in Caenorhabditis elegans. Nature 410, 227-230. doi: $10.1038 / 35065638$

Van Laar, V. S., and Berman, S. B. (2009). Mitochondrial dynamics in Parkinson's disease. Exp. Neurol. 218, 247-256. doi: 10.1016/j.expneurol.2009. 03.019

Vakhrusheva, O., Smolka, C., Gajawada, P., Kostin, S., Boettger, T., Kubin, T., et al. (2008). Sirt7 increases stress resistance of cardiomyocytes and prevents apoptosis and inflammatory cardiomyopathy in mice. Circ. Res. 102, 703-710. doi: 10.1161/CIRCRESAHA.107.164558

Verma, R., Ritzel, R. M., Crapser, J., Friedler, B. D., and McCullough, L. D. (2018). Evaluation of the neuroprotective effect of Sirt3 in experimental stroke. Transl. Stroke Res. doi: 10.1007/s12975-017-0603-x [Epub ahead of print].

Waitt, A. E., Reed, L., Ransom, B. R., and Brown, A. M. (2017). Emerging roles for glycogen in the CNS. Front. Mol. Neurosci. 10:73. doi: 10.3389/fnmol.2017. 00073

Wang, X., Su, B., Lee, H. G., Li, X., Perry, G., Smith, M. A., et al. (2009). Impaired balance of mitochondrial fission and fusion in Alzheimer's disease. J. Neurosci. 29, 9090-9103. doi: 10.1523/JNEUROSCI.1357-09.2009

Weir, H. J., Murray, T. K., Kehoe, P. G., Love, S., Verdin, E. M., O’Neill, M. J., et al. (2012). CNS SIRT3 expression is altered by reactive oxygen species and in Alzheimer's disease. PLoS One 7:e48225. doi: 10.1371/journal.pone. 0048225

White, H., and Venkatesh, B. (2011). Clinical review: ketones and brain injury. Crit. Care 15:219. doi: 10.1186/cc10020

Wood, J. G., Rogina, B., Lavu, S., Howitz, K., Helfand, S. L., Tatar, M., et al. (2004). Sirtuin activators mimic caloric restriction and delay ageing in metazoans. Nature 430, 686-689. doi: 10.1038/nature02789

Wu, Y. T., Lee, H. C., Liao, C. C., and Wei, Y. H. (2013). Regulation of mitochondrial $\mathrm{F}_{o} \mathrm{~F}_{1}$ ATPase activity by Sirt3-catalyzed deacetylation and its deficiency in human cells harboring 4977bp deletion of mitochondrial DNA. Biochim. Biophys. Acta 1832, 216-227. doi: 10.1016/j.bbadis.2012. 10.002

Wu, S., Zhou, F., Zhang, Z., and Xing, D. (2011). Mitochondrial oxidative stress causes mitochondrial fragmentation via differential modulation of 
mitochondrial fission-fusion proteins. FEBS J. 278, 941-954. doi: 10.1111/j. 1742-4658.2011.08010.x

Xavier, J. M., Rodrigues, C. M., and Solá, S. (2016). Mitochondria: major regulators of neural development. Neuroscientist 22, 346-358. doi: 10.1177/1073858415585472

Yamashita, H., Kaneyuki, T., and Tagawa, K. (2001). Production of acetate in the liver and its utilization in peripheral tissues. Biochim. Biophys. Acta 1532, 79-87. doi: 10.1016/s1388-1981(01)00117-2

Yang, Y., Cimen, H., Han, M. J., Shi, T., Deng, J. H., Koc, H., et al. (2010). NAD+dependent deacetylase SIRT3 regulates mitochondrial protein synthesis by deacetylation of the ribosomal protein MRPL10. J. Biol. Chem. 285, 7417-7429. doi: 10.1074/jbc.M109.053421

Yang, X., Geng, K., Zhang, J., Zhang, Y., Shao, J., and Xia, W. (2017). Sirt3 mediates the inhibitory effect of adjudin on astrocyte activation and glial scar formation following ischemic stroke. Front. Pharmacol. 8:943. doi: 10.3389/fphar.2017. 00943

Yang, W., Zou, Y., Zhang, M., Zhao, N., Tian, Q., Gu, M., et al. (2015). Mitochondrial Sirt3 expression is decreased in APP/PS1 double transgenic mouse model of Alzheimer's disease. Neurochem. Res. 40, 1576-1582. doi: 10.1007/s11064-015-1630-1

Yin, J., Han, P., Song, M., Nielse, M., Beach, G. T., Serrano, G. E., et al. (2018). Amyloid- $\beta$ increases tau by mediating sirtuin 3 in Alzheimer's disease. Mol. Neurobiol. doi: 10.1007/s12035-018-0977-0 [Epub ahead of print].

Yu, W., Dittenhafer-Reed, K. E., and Denu, J. M. (2012). SIRT3 protein deacetylates isocitrate dehydrogenase 2 (IDH2) and regulates mitochondrial redox status. J. Biol. Chem. 287, 14078-14086. doi: 10.1074/jbc.m112.355206

Zeng, L., Yang, Y., Hu, Y., Sun, Y., Du, Z., Xie, Z., et al. (2014). Age-related decrease in the mitochondrial sirtuin deacetylase Sirt3 expression associated with ROS accumulation in the auditory cortex of the mimetic aging rat model. PLoS One 9:e88019. doi: 10.1371/journal.pone.0088019
Zhang, Y., Bharathi, S. S., Rardin, M. J., Uppala, R., Verdin, E., Gibson, B. W., et al. (2015). SIRT3 and SIRT5 regulate the enzyme activity and cardiolipin binding of very long-chain acyl-CoA dehydrogenase. PLoS One 10:e122297. doi: 10.1371/journal.pone.0122297

Zhang, J. Y., Deng, Y. N., Zhang, M., Su, H., and Qu, Q. M. (2016). SIRT3 acts as a neuroprotective agent in rotenone-induced parkinson cell model. Neurochem. Res. 41, 1761-1773. doi: 10.1007/s11064-016-1892-2

Zhao, H. C., Ding, T., Ren, Y., Li, T. J., Li, R., Fan, Y., et al. (2016). Role of Sirt3 in mitochondrial biogenesis and developmental competence of human in vitro matured oocytes. Hum. Reprod. 31, 607-622. doi: 10.1093/humrep/dev345

Zhao, K., Zhou, Y., Qiao, C., Ni, T., Li, Z., Wang, X., et al. (2015). Oroxylin A promotes PTEN-mediated negative regulation of MDM2 transcription via SIRT3-mediated deacetylation to stabilize p53 and inhibit glycolysis in wt-p53 cancer cells. J. Hematol. Oncol. 8:41. doi: 10.1186/s13045-0150137-1

Conflict of Interest Statement: The authors declare that the research was conducted in the absence of any commercial or financial relationships that could be construed as a potential conflict of interest.

The reviewer LET declared a past co-authorship with one of the authors JE to the handling Editor.

Copyright (c) 2018 Sidorova-Darmos, Sommer and Eubanks. This is an open-access article distributed under the terms of the Creative Commons Attribution License (CC BY). The use, distribution or reproduction in other forums is permitted, provided the original author(s) and the copyright owner(s) are credited and that the original publication in this journal is cited, in accordance with accepted academic practice. No use, distribution or reproduction is permitted which does not comply with these terms. 